\title{
Review
}

Midya Parto, Yuzhou G. N. Liu, Babak Bahari, Mercedeh Khajavikhan and

Demetrios N. Christodoulides*

\section{Non-Hermitian and topological photonics: optics at an exceptional point}

https://doi.org/10.1515/nanoph-2020-0434

Received July 29, 2020; accepted September 4, 2020;

published online October 2, 2020

\begin{abstract}
In the past few years, concepts from nonHermitian (NH) physics, originally developed within the context of quantum field theories, have been successfully deployed over a wide range of physical settings where wave dynamics are known to play a key role. In optics, a special class of NH Hamiltonians - which respects paritytime symmetry - has been intensely pursued along several fronts. What makes this family of systems so intriguing is the prospect of phase transitions and $\mathrm{NH}$ singularities that can in turn lead to a plethora of counterintuitive phenomena. Quite recently, these ideas have permeated several other fields of science and technology in a quest to achieve new behaviors and functionalities in nonconservative environments that would have otherwise been impossible in standard Hermitian arrangements. Here, we provide an overview of recent advancements in these emerging fields, with emphasis on photonic $\mathrm{NH}$ platforms, exceptional point dynamics, and the very promising interplay between non-Hermiticity and topological physics.
\end{abstract}

Keywords: exceptional points; non-Hermitian physics; PT symmetry; topological photonics.

\footnotetext{
*Corresponding author: Demetrios N. Christodoulides, CREOL, College of Optics and Photonics, University of Central Florida, Orando, 4304 Scorpius St, Orlando, USA, E-mail: demetri@creol.ucf.edu. https://orcid.org/0000-0003-3630-7234

Midya Parto, CREOL, College of Optics and Photonics, University of Central Florida, 4304 Scorpius St, Orlando, Florida, USA. https:// orcid.org/0000-0003-2100-5671

Yuzhou G. N. Liu and Babak Bahari, Viterbi School of Engineering, University of Southern California, 3737 Watt Way, Los Angeles, Los Angeles, California, USA

Mercedeh Khajavikhan, Viterbi School of Engineering, University of Southern California, 3737 Watt Way, Los Angeles, Los Angeles, California, USA; CREOL, College of Optics and Photonics, University of Central Florida, 4304 Scorpius St, Orlando, Florida, USA. https:// orcid.org/0000-0002-7091-1470
}

\section{Introduction}

Quantum mechanics dictates that every observable should be described by means of a self-adjoint or Hermitian operator. In this respect, the Hamiltonian of a system being no exception to this rule - must exhibit real eigenenergies and orthogonal eigenstates, attributes necessary for unitary evolution and conservation of probability. However, while this universal conservation principle does apply for a closed system as a whole, there is nothing to exclude the possibility of energy exchange among its subsystems. When considered individually, each subsystem can see an overall growth or decay in energy or probability norm - aspects that can phenomenologically be accounted for through the adoption of complex energy eigenvalues. Indeed, approaching quantum mechanical phenomena from such a "nonconservative" standpoint can be traced back to the early studies of Gamow [1] on particle decay or other contributions on neutron scattering [2]. On the other hand, in many classical settings like optics, nonHermiticity is not always welcome. In particular, energy/ power dissipation has been traditionally considered something undesirable, an aspect to be mitigated at all costs (typically through the use of amplification) in order to maintain the performance metrics of a device or a system. At this point, one may naturally ask as to whether loss is always a problem. If not, is there a strategy to use it judiciously in order to attain new degrees of freedom?

A radical change in the way we perceive many of the aforementioned aspects occurred when Bender and Boettcher [3] realized that a large class of non-Hermitian (NH) Hamiltonians can exhibit entirely real spectra, provided that they commute with the parity-time (PT) operator. Here, the parity operator $P$ represents a reflection in the coordinate space with respect to the origin, while $\mathrm{T}$ signifies the time-reversal operator. This rather counterintuitive result suggests that a PT-symmetric Hamiltonian can display altogether real eigenvalues whenever its pertinent NH parameters lie in the PT symmetry unbroken phase. On the other hand, once the non-Hermiticity 
parameter exceeds a certain critical threshold, the eigenstates spontaneously break PT symmetry, thus entering into a PT broken phase. This marks the onset of a phase transition that entails NH degeneracies, better known as exceptional points (EPs) [4]. In addition, the eigenfunctions associated with these $\mathrm{NH}$ operators are no longer orthogonal with each other; instead, they are now skewed. Starting from these premises, one can then directly show that a necessary (albeit not sufficient) condition for a $\mathrm{NH}$ Hamiltonian to be PT symmetric is that its complex potential should satisfy $V(\vec{r})=V^{*}(-\vec{r})$ [3]. From here, one can conclude that the real part of the potential must be symmetric with respect to the origin, while the imaginary component must be antisymmetric.

While the physical ramifications of the aforementioned mathematical findings remained for several years a matter of debate, a series of subsequent studies indicated that optics could provide instead an ideal test bed to realize and experimentally investigate the implications of PT symmetry and $\mathrm{NH}$ physics in actual settings (see Figure 1AD) [5-10]. After all, in photonic arrangements, the refractive index profile plays the role of the potential in quantum mechanics. Consequently, optical PT symmetry can be readily established by judiciously distributing the gain and loss in such a way that the refractive index profile is an even function of position while the optical gain/loss emerges as an odd function in the spatial coordinates. These early studies incited a flurry of research activities in many and diverse fields such as microwaves [11], electronics [12], mechanics [13], optomechanics [14, 15], acoustics [16, 17], atomic lattices [18-20], etc., all aiming to harness the very characteristics of PT symmetry and EPs.

A distinctive feature of optical arrangements is the possibility of controlling both the real and imaginary parts of the electromagnetic permittivity in an independent manner, without being over-restricted by the KramersKronig relations. In this regard, photonic platforms can host a multitude of fascinating wave phenomena that solely arise owing to a synergy between PT symmetry and EPs on the one hand and Hermitian symmetries on the other hand. A profound example of such fruitful interactions is the newly emerging field of $\mathrm{NH}$ topological photonics. Topological notions originally arose within the context of condensed matter physics after the discovery of topological insulators (TIs), where electron conduction was found to be prohibited in the bulk while it can take place in the periphery of a material via topologically protected unidirectional edge states [21-23]. These developments in turn inspired further research endeavors in employing topological notions in optical arrangements, which led to the observation of unidirectional transport and robust topological edge modes in coupled resonators and waveguide lattices [24-26]. Unlike in early efforts where the emphasis was on conservative optical systems, quite recently, there has been an ever-growing interest in expanding these concepts into $\mathrm{NH}$ photonic structures.

In this review article, we focus on novel phenomena in photonics that are enabled by the synergies among

\section{A}

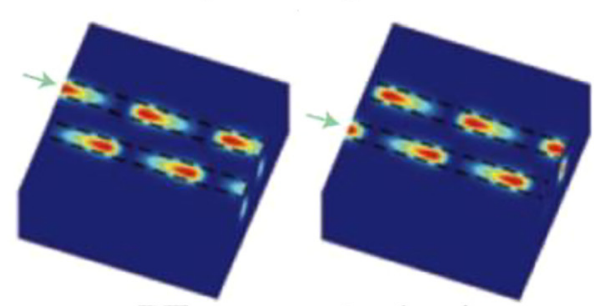

PT-symmetry broken

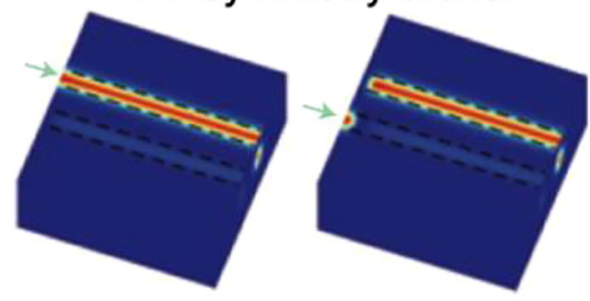

B

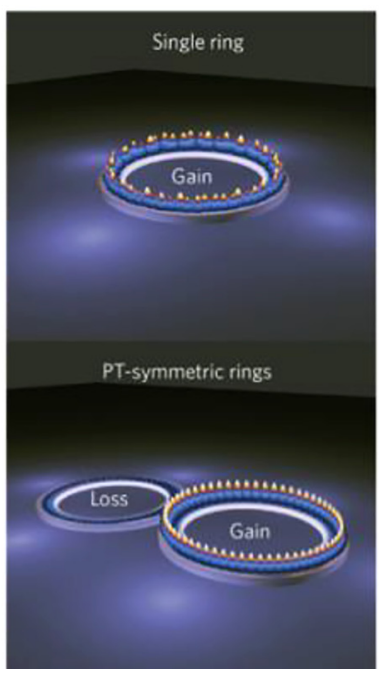

C
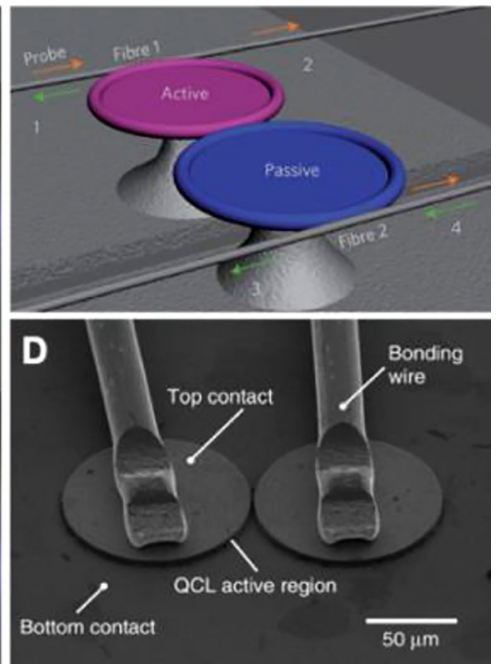

Figure 1: PT symmetry in optics.

(A) Different regimes associated with parity-time (PT)-symmetry breaking manifested in light propagation dynamics within a non-Hermitian (NH) waveguide coupler [9]. (B) Enforcing single-mode lasing in a pair of PT-symmetric microring resonators, each supporting a multitude of lasing states in isolation [55]. (C) A schematic representation of whispering-gallery-mode PT-symmetric microtoroid cavities [48]. (D) Coupled quantum-cascade-laser (QCL) arrangement used to observe pump-induced suppression and revival of lasing [50]. 
non-Hermiticity, topology, and various other types of symmetries. In Section 2, we discuss PT symmetry and its various realizations in nonconservative optical structures. Section 3 focuses on the physics of EPs and the exotic effects displayed by photonic systems that happen to operate in the vicinity of such $\mathrm{NH}$ degeneracies. Section 4 is devoted to topological concepts and their manifestations in connection with non-Hermiticity in the presence of certain classes of symmetries. Finally, Section 5 provides a summary along with an outlook in this general area of research.

\section{Non-Hermitian photonics and PT symmetry}

As mentioned earlier, an important class of NH Hamiltonians that are capable of exhibiting entirely real eigenvalues is that respecting PT symmetry. In optics, PT symmetry can be established by judiciously distributing gain and loss, processes that are readily accessible in a wide variety of optical platforms ranging from bulk cavities and optical fiber amplifiers to on-chip photonic circuits (Figure 1A). In this regard, optics provides a fertile ground for exploring $\mathrm{NH}$ phenomena and their ensuing effects when considered in conjunction with other conservative effects associated with light dynamics. This, in turn, has led to a paradigm shift in molding the flow of light, which was thus far traditionally limited to only shaping the refractive index distribution, starting from the early development of the first lenses and reaching the modern era that witnessed the advent of more sophisticated optical systems like photonic crystal fibers and metamaterials [27-30]. In this section, we focus on some of the exotic behaviors resulting from the introduction of nonHermiticity and PT symmetry in optical arrangements.

It should be noted that NH effects in photonic settings are not limited to systems exhibiting optical absorption and/or amplification [31-37]. Such phenomena can also arise, for instance, owing to an energy exchange between metastable guided states and leaky modes in optical fibers or whenever a conservative subsystem is open [38-41].

\subsection{Lasers and non-Hermitian symmetry breaking}

Lasers provide an ideal test bed for studying some of the ramifications of non-Hermiticity. Both gain and loss are indispensable components of any light system, especially when there is a need to overcome optical absorption through the use of amplification. It therefore comes as no surprise that signatures of non-Hermiticity had already been considered in early efforts on lasers, manifested, for example, in mode nonorthogonalities that are known to lead to the Petermann K-factor enhancement [42-44] in the fundamental Schawlow-Townes linewidth [45] of a laser cavity. Nevertheless, until very recently, almost all efforts in designing laser resonators were aimed at lowering the dissipation, something that was traditionally deemed detrimental to the performance of these devices.

The recent developments in NH optics and PT symmetry have provided a systematic framework to explore exotic regimes of behavior in laser systems. Perhaps, the most archetypical example is a pair of two coupled identical cavities, with one being subjected to gain while the other being subjected to an equal amount of loss. Following the previous discussion, this arrangement is PT symmetric, given that the gain/loss distribution is antisymmetric while index-wise is even [46-48]. In the vicinity of the PT phase transition, i.e., at the EP, this structure was shown to exhibit a counterintuitive pump-induced suppression and revival of lasing (Figure 1D) [49-52]. Variations of this behavior have also been demonstrated in a phonon laser arrangement [14], where substantial linewidth enhancement at the EP was found to occur [53]. This latter effect is a by-product of the collapsing supermodes at the degeneracy point, an extreme case of the mode nonorthogonality mentioned earlier.

On many occasions, a primary goal in laser design is to enforce single-mode operation to achieve a coherent, highquality output. A general trend in fulfilling this requirement is to use miniature semiconductor lasers. However, the inhomogeneously broadened gain bandwidth in such semiconductor-based active materials can easily span a wavelength range that is many times larger than the free spectral range associated with a typical microring cavity, hence eluding the aforementioned goal of exclusively lasing in a single longitudinal mode. One way to mitigate this issue is to use dispersive elements similar to those used, for example, in distributed feedback lasers. An alternative route to accomplish this goal is to exploit the PT symmetry in the underlying cavity structure. In this scenario, by adjusting the pump levels in the system, one can ensure that PT symmetry is broken for only one longitudinal mode, while the other supported states are kept below the PT threshold and hence exhibit real eigenvalues with no amplification. This in turn ensures single-mode operation of the device while all the other principal attributes of the laser cavity remain intact. This scheme has been lately demonstrated in microring lasers (involving 
whispering gallery modes) without any compromise in terms of slope efficiency or threshold pump intensities (see Figure 1B, C) [54-57]. PT-symmetric lasers have also been demonstrated in various other platforms such as electrically pumped integrated arrangements $[58,59]$ and optical fibers [60]. A similar approach can be applied to achieve single-frequency lasing through the use of EPs, an aspect demonstrated in dark-state lasers [61, 62].

Non-Hermiticity and loss management in active lattices is shown to be an effective tool for implementing various spin Hamiltonians in an optical platform [63-67]. In these schemes, the loss that vectorial electromagnetic modes experience on the interface of metallic nanocavities provides an effective way to establish the ferromagnetic and antiferromagnetic type of exchanges. Based on such arrangements, large arrays of nanolasers have been realized, which emit in a single mode and with a desired topological singularity $[66,67]$. In laser arrays, the interplay between supersymmetry (SUSY) and non-Hermiticity has also been shown to result in single spatial mode operation. In this regard, a waveguide array subject to gain is coupled to a lossy superpartner. With the exception of the fundamental mode that has no counterpart in the partner array, all other modes of the main array share the same eigenfrequencies with those in the superpartner. By adjusting the gain-loss contrast between the two arrays, one can keep all the higher order modes of the array below the PT symmetry breaking point, thus allowing the fundamental mode to experience gain and subsequently lase $[68,69]$.

On-chip single-mode microlasers based on EPs have so far found applications in generating states with prespecified orbital angular momentum (OAM) on a chip. Typically, the counterpropagating modes within a microring laser cavity form a degenerate pair which tend to simultaneously lase together once the gain exceeds threshold. This precludes a direct generation of a vortex beam with nonzero topological charge since the two opposite azimuthal mode numbers tend to cancel each other. NH schemes based on EPs have recently provided an elegant method to selectively extract only one chiral mode in such lasers [52, 60-72]. This class of devices can emit in a tunable OAM order while operating in a broadband fashion. Another interesting effect that is closely tied to the aforementioned $\mathrm{NH}$ aspects is the so-called coherent perfect absorption, where a coherent monochromatic light input is entirely absorbed by a lossy medium [73, 74]. In this respect, a coherent perfect absorber (CPA) acts as a time-reversed version of a laser. Interestingly, it has been shown that a PT-symmetric cavity can simultaneously behave as a laser and a CPA at the same frequency $[75,76]$.

\subsection{PT-symmetric metamaterials and non-Hermitian cloaking}

The past two decades have witnessed considerable research efforts that are geared to develop artificial materials, tailored to display properties that are not found in nature. Yet, until recently, such electromagnetic metamaterials have almost exclusively relied only on modifying the real permittivities and permeabilities associated with the material elements [30]. The recent developments in $\mathrm{NH}$ photonics have opened up new avenues in exploring lightmatter interactions in the entire complex plane of the material constitutive parameters.

In the linear Hermitian domain, a one-dimensional (1D) grating exhibits transmission and reflection properties that are typically independent from the direction the light impinges upon the structure. On the other hand, this feature can be violated in a NH grating. In particular, one can establish gain and loss regions within the unit cell of a grating in such a way that the reflection coefficients are direction dependent (Figure 2A) [77]. This asymmetric wave propagation becomes mostly pronounced at the EP, where light can propagate completely without reflection from one side while exhibiting strong reflection in the opposite direction, a process leading to unidirectional invisibility. Interestingly, because the system is not conservative in this case, the reflection values can greatly exceed unity [77]. Unidirectional invisibility was recently observed in various platforms including photonic mesh lattices (see Figure 2B) $[78,79]$, passive silicon periodic nanowires [80], multilayer $\mathrm{Si} / \mathrm{SiO}_{2}$ structures [81], organic films [82], and electroacoustic resonators [17].

The peculiar wave transport in NH systems is known to result in other unconventional effects such as unidirectional cloaking $[83,84]$. This could be achieved via a PT-symmetric surface that surrounds an object with an arbitrary size. In such a scenario, a lossy part in the surface is designed to entirely absorb the incoming wave while a corresponding active segment can reemit the same amount of power impinging on the object. The result is a broadband cloaking device which benefits from relaxed design constraints owing to its active architecture. Another relevant aspect to this discussion are the so-called "constant intensity waves”. Typically, in a lossless medium, an electromagnetic wave (like a plane wave) can remain invariant during propagation when the propagation space is homogeneous. Any inhomogeneity such as an obstacle would inevitably cause reflections and scattering, which disturb the original uniform wavefront. In sharp contrast to this Hermitian picture, by introducing gain and loss in a 
A

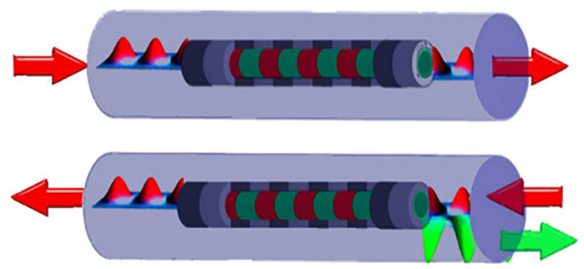

C

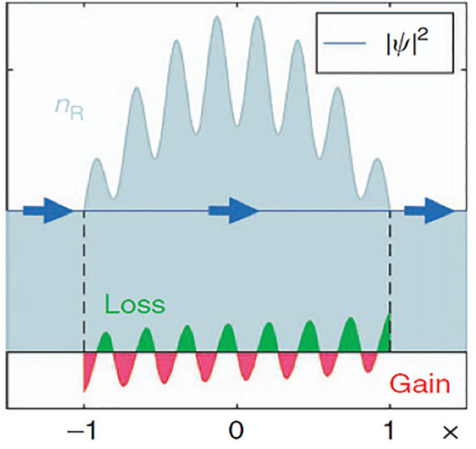

B

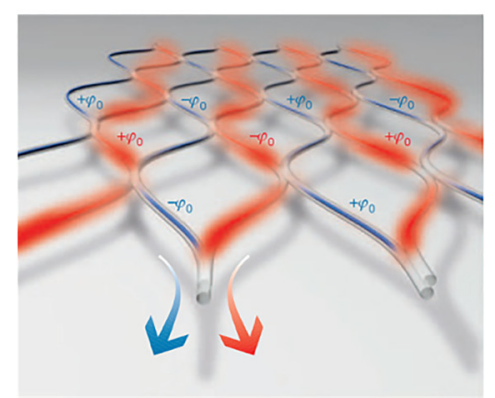

D

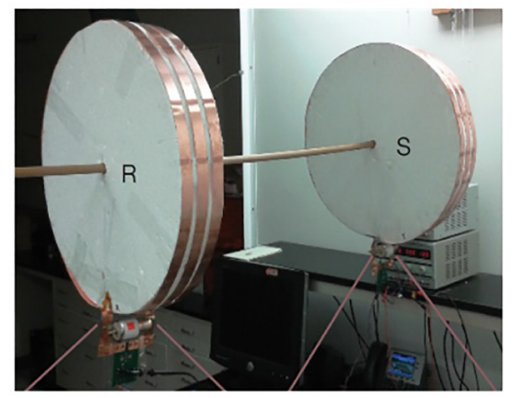

Figure 2: Unidirectional invisibility and parity-time (PT)-symmetry.

(A) A schematic of a PT-symmetric grating that exhibits unidirectional invisibility [77]. (B) Photonic mesh lattices utilized to observe exceptional points [78]. (C) Plane wave propagation in a scattering medium with appropriately patterned gain and loss resulting in a constant intensity wave profile throughout the entire structure [86].

(D) Experimental setup employed to demonstrate efficient wireless power transfer in a nonlinear PT-symmetric arrangement [99]. general class of nonuniform potentials, one can construct a constant-intensity wave solution, even systems with random index distributions (Figure 2C) [85, 86].

Other types of PT-symmetric and complex lattices have been studied and shown to behave quite differently from their Hermitian counterparts. For instance, beam dynamics in a PT-symmetric optical lattice can display band merging effects in the associated complex band structure with EPs emerging in the Bloch momentum space [6]. In addition, even though the material parameters in such lattices are isotropic, the light propagation through the array could exhibit double refraction. This latter unusual behavior stems from the skewedness in the associated FloquetBloch modes of the structure. Nonreciprocal Bloch oscillations is yet another peculiar phenomenon that can occur in such complex crystals with no analog in Hermitian arrays [87]. Other works have studied $\mathrm{NH}$ symmetry breaking in graphene-like lattices [88], PT Talbot revivals [89], and Anderson localization in disordered PT-symmetric arrangements [90].

Extensions of these ideas have been lately pursued in PT-symmetric metasurfaces. It has been shown that negative refraction and planar focusing could be achieved in such $\mathrm{NH}$ sheets without engaging negativeindex metamaterials or phase-conjugating surfaces [91-93]. Such structures could enable loss-free all-angle negative refraction and planar lenses in free space. PT-symmetric phase transitions have also been studied in the polarization space of a complex metasurface, in which the eigenstates eventually collapse on each other on the Poincaré sphere when the system reaches an EP [94]. Similar ideas have been exploited to diffract light with asymmetric diffraction orders using a deformed honeycomb metasurface with a diatomic Bravais-lattice topology [95].

\subsection{Nonlinear effects in non-Hermitian systems}

Nonlinear effects are an integral part of many optical platforms used for implementing NH Hamiltonians. For instance, gain saturation nonlinearities in active materials are known to be responsible for stabilizing laser oscillators. It is therefore natural to investigate the interplay between nonlinearity and non-Hermiticity and the prospect for $\mathrm{NH}$ phases such as PT symmetry under such conditions. In this regard, it has been theoretically predicted that optical Kerr nonlinearities can reverse PT-symmetric phases, i.e., transforming a linear system in the PT-unbroken phase to a nonlinear one with a broken symmetry and vice versa [96]. Such nonlinearly induced phase transitions were later shown to also hold in the case of gain saturation nonlinearities and were successfully observed in coupled semiconductor microring lasers [97, 98]. Nevertheless, even in the presence of these nonlinear processes, the 
eigenmodes of the system still retain their corresponding forms in the linear regime [97]. Interestingly, nonlinear processes can have practical implications in active environments that go beyond lasers. For instance, it has been shown that robust wireless power transfer could be accomplished through the realization of a nonlinear PT-symmetric circuit (Figure 2D) [99]. In this scenario, the nonlinearity guarantees that the system remains in the PT-unbroken phase for strong enough couplings, a feature that allows for a wide range of accessible distances between the source and the receiver. This in turn eliminates the need for constant tunings of the corresponding resonators that is otherwise necessary to attain efficient power transfer.

The impact of non-Hermiticity on nonlinear processes such as that of three- and four-wave mixing has also been investigated. In this vein, non-Hermiticity could assist phase-matching in optical parametric amplifiers when a Hermitian system cannot satisfy these conditions [100, 101]. Such techniques could facilitate parametric amplification in long-wavelength regimes using on-chip semiconductor arrangements with large nonlinearities. In addition, the interplay between nonlinearity and non-Hermiticity in a PT-symmetric optical coupler can lead to interesting functionalities such as optical switching [102] and selective parametric amplification in the spectral domain [103]. Similar concepts have also been considered in optomechanical settings, where the nonlinearity is induced by mechanical vibrations [15]. Along different lines, it has been shown that PT symmetry can be established solely by optical nonlinear processes, without requiring active elements [104]. Such nonlinearity-induced PT effects are instead enabled by parametric gain in a three-wave mixing scenario.

Another interesting function emerging from the combined effects of optical gain and loss is providing additional tools to control light transport in active nonlinear environments $[7,105]$. An important class of such $\mathrm{NH}$ systems satisfies PT-symmetric conditions with balanced amplification and decay [106-108]. In this vein, PT-symmetry plays a crucial role in determining the stability regimes of nonlinear excitations such as optical solitons in such settings. For instance, it has been predicted and experimentally observed that contrary to other $\mathrm{NH}$ nonlinear systems wherein self-trapped states emerge as fixed points in the parameter space, discrete PT solitons can form a continuous family of solutions [79, 108]. Moreover, the synergy between non-Hermiticity and nonlinearity has been deployed to demonstrate unidirectional light transport $[109,110]$. Such structures could lead to new approaches in developing all-dielectric on-chip optical components such as circulators.

\section{Exceptional points in optics}

An EP is a special type of degeneracy in the complex parameter space of a non-Hermitian Hamiltonian. The most profound characteristic of an EP that distinguishes it from a regular Hermitian degeneracy is the fact that not only the eigenvalues degenerately coalesce at this point but also their corresponding eigenvectors simultaneously collapse on each other-leading to an abrupt reduction in dimensionality. In general, an EP is said to be of the order $\mathrm{N}$, if at the same time, $\mathrm{N}$ eigenvalues and their respective eigenvectors fuse with each other at this $\mathrm{NH}$ degeneracy $[111,112]$. This exotic property in turn leads into an array of fascinating effects that are unique to $\mathrm{NH}$ systems operating at or close to such singularities (see Figure 3A-D). In this section, we discuss some of these unconventional aspects.

\subsection{Enhancement effects around EPs}

Over the years, optics has provided some of the most accurate metrology tools for a variety of sensing applications. These devices range from optical gyroscopes and tachometers to chemical and biomedical sensors, to name a few. In this regard, optical resonators have been widely utilized for such purposes mainly due to their ability to provide a strong interaction between the light field and the sensing target. In recent years, the development of ultralow-loss microtoroids [113] and low loss silicon microresonators [114] has sparked a great deal of interest in implementing photonic sensors on a chip.

In spite of their remarkable performance, standard microcavity resonators (characterized by a set of orthogonal modes) are still limited within the bounds imposed by their Hermitian nature. This aspect can be better understood from the perspective of standard perturbation theory. As is well known, if a Hermitian system is perturbed to order $\varepsilon$, then its eigenvalues $\lambda$ can be obtained from the familiar power series $\lambda=\lambda_{0}+\lambda_{1} \varepsilon+\lambda_{2} \varepsilon^{2}+\cdots$, from where one can quickly conclude that the response of any Hermitian arrangement is at best linear with respect to the disturbance $\varepsilon$. In contrast, if a NH system is biased at an EP of order $N$, once perturbed to order $\varepsilon$, its associated eigenvalues instead follow a Newton-Puiseux series $\lambda=\lambda_{0}+\lambda_{1} \varepsilon^{1 / N}+\lambda_{2} \varepsilon^{1 / N}+\cdots$ In other words, when an $\mathrm{NH}$ configuration is placed at an EP of order $N$, its first order response is now expected to vary according to $\varepsilon^{1 / N}$. Given 

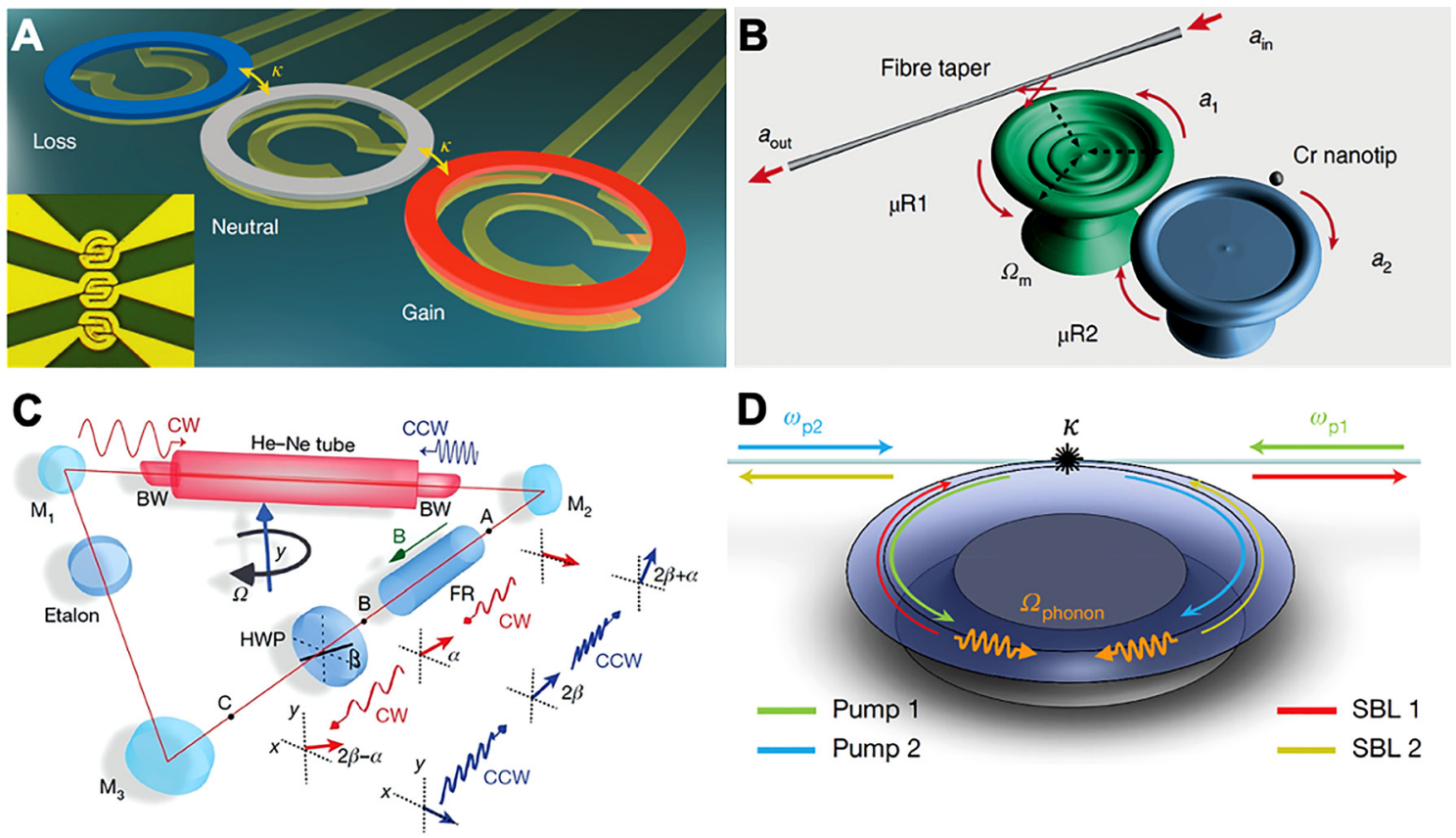

Figure 3: Enhanced sensitivity using exceptional points (EPs).

(A) Coupled microring lasers equipped with microheaters used to realize a third-order EP [118]. (B) A coupled microtoroid phonon laser operating at an exceptional point [53]. (C, D) Enhanced Sagnac effect in EP-based gyroscopes implemented in a ring laser gyroscope [121] and a Brillouin laser cavity [122], respectively.

that for small $\varepsilon, \varepsilon^{1 / N} \gg \varepsilon$, then it is straightforward to deduce that an $\mathrm{NH}$ arrangement (at an $\mathrm{EP}$ ) can react considerably more drastically than its Hermitian counterpart. Intuitively, this distinctive behavior stems from the abrupt phase transition associated with the reduction in the dimensionality of the corresponding eigenspace. As a result, when dealing with small input signals, the response of EP-based structures can be boosted by orders of magnitude.

Lately, this magnified response provided by EPs has been employed to realize optical sensors with enhanced performance. This can be achieved for example by incorporating two or more scatterers around a passive microcavity which establishes an EP involving the two counterpropagating whispering-gallery modes within the structure $[115,116]$. Theoretical results suggest that such an arrangement could enhance single-particle detection sensitivity by a factor of seven as compared to an isolated microcavity [117]. This concept was later demonstrated experimentally both in active and passive photonic platforms. In an active scenario, coupled microring lasers in binary and ternary PT symmetric photonic molecule arrangements have been used to demonstrate second- and third-order EPs, respectively (Figure 3A) [118]. In this case, more than an order of magnitude sensitivity enhancement has been reported which can be boosted even further by the amplitude of the gain present in the system. An alternative realization involves a passive single whispering-gallery optical microresonator which is brought to a second-order EP by using two lossy Rayleigh scatterers [119]. A third scatterer is then used as the target object to be detected which causes a frequency splitting in the complex plane.

From a practical perspective, the augmented response enabled via $\mathrm{NH}$ degeneracies has found direct applications in Sagnac-based gyroscopes [120], which can be realized by retrofitting a helium-neon ring laser gyroscope ( $\mathrm{He}-\mathrm{Ne}$ RLG) with a Faraday rotator and a half-wave plate (see Figure 3C) [121]. Combined with Brewster windows surrounding the gain tube, these components can then introduce a differential gain/loss contrast in the system, necessary for establishing an EP. Experimental measurements on such devices indicate a square-root dependence of the frequency response on the gyration speed, in contrast to the linear behavior in a standard arrangement, leading to a twenty-fold enhancement in sensitivity. A different implementation of an EP-enhanced Sagnac effect has been experimentally demonstrated using a microring Brillouin laser (Figure 3D) [122]. By incorporating a fiber taper in the vicinity of the microring, a dissipative coupling takes place between the two counterpropagating modes involved. This in turn induces a second-order EP at a critical pumpdetuning frequency, resulting in a four-fold increase in the Sagnac scale factor while allowing for measuring rotations of approximately one revolution per hour. 
Interestingly, enhancement effects in systems involving EPs are not limited to sensing [123, 124]. In fact, it has been suggested that these $\mathrm{NH}$ degeneracies can considerably modify light-matter interactions within an active structure, leading into substantially higher spontaneous emission rates (Figure 3B) [125]. As opposed to traditional spontaneous emission theories that lead to "infinite" values at an EP, an analysis that consistently takes into account the local density of states predicts a bounded response. Specifically, in passive structures involving second-order EP2 degeneracies, this bound is found to be a four-fold enhancement in the associated spontaneous emission. Another consequence emerging from the presence of an EP in such settings is the emission lineshape itself which can deviate from the Lorentzian profile, thus resulting in a nonlinear scaling of the spontaneous emission with the associated resonance quality factors. Similar studies have also been conducted within the context of $\mathrm{NH}$ photonic crystals hosting third-order EP3 degeneracies where an eight-fold enhancement is expected [126]. These results can also be generalized to EPs of higherorder $N$ inducing larger emissions by a factor of $\sqrt{N^{3}}$ [126].

The fact that EPs are highly sensitive to changes in their environment is not always desirable. For instance, an EP sensor can also be excessively vulnerable to fabrication errors and imperfections that are inevitable in experiments. In this respect, exceptional surfaces have been suggested as a possible avenue to combine the robustness required for practical applications with the characteristic sensitivity offered by EPs [127]. One way to achieve this is to introduce a unidirectional coupling between the counterpropagating modes of a microring cavity. The resulting NH Hamiltonian describing the system features an exceptional surface in the parameter space. In this case, undesired perturbations such as random variations in the coupling coefficients or the resonant frequency of the cavity cause the system to move across this exceptional surface, thus maintaining the useful properties of an EP. On the other hand, when external scatterers start to perturb the cavity, the structure is promptly pushed out of the exceptional surface as a result of the bidirectional coupling that is now induced between the counterpropagating modes. In this latter case, an amplified response could be measured in the spectral splitting of the device. The exceptional surfaces mentioned here are also known to arise in other photonic arrangements such as three-dimensional (3D) PT-symmetric photonic crystals $[71,128]$.

The prospect of using EPs for optical sensing has recently prompted an investigation of practical aspects associated with these applications such as noise figures. In particular, because boosting the input signal is typically accompanied by an unwanted enhancement of various noise sources, it is not immediately clear if EP-based sensors could offer a superior signal-to-noise ratio (SNR) [129, 130]. To this end, the role of classical noise in the form of mesoscopic fluctuations on the spectral and temporal behavior of resonator-based arrangements operating near an EP has recently been studied [131]. In these configurations, the presence of noise results in frequency detuning among the constituent resonant entities, which in turn modifies the conditions for reaching an EP. Moreover, statistical averaging of the aforementioned fluctuations could smear the spectral features, hence downgrading the effective sensitivity of EP-based sensors to noise-limited values. Along different lines, the performance of EP sensing can be analyzed from the point of view of quantum noise theory [132]. In this vein, it has been shown that by using the quantum Fisher information one could obtain a lower bound for the SNR associated with an EP sensor. These theoretical results predict that by implementing an EP amplifier near the lasing threshold in conjunction with a heterodyne detection scheme, an improved SNR performance as compared with Hermitian sensors can be achieved.

\subsection{Encircling EPs and mode conversion}

A remarkable behavior of $\mathrm{NH}$ degeneracies is related to the dynamical behavior of their associated Hamiltonian. In Hermitian settings, a cyclic evolution that occurs in an adiabatic manner tends to preserve an eigenstate of the system, apart from a geometric phase factor [133]. This picture could completely break down in the case of $\mathrm{NH}$ structures involving an EP. A possible scenario in this regard is when the system undergoes a cyclic evolution in a quasi-static fashion. In this case, the instantaneous eigenstates will swap with each other at the end of a single cycle, apart from acquiring a geometric phase [134, 135]. This peculiar behavior can be attributed to the geometry of the intersecting complex Riemann sheets unique to nonconservative systems and has been experimentally observed in microwave [136] and optical cavities [137] as well as exciton-polaritonic arrangements [138]. Alternatively, the NH Hamiltonian may change in such a way that the EP encirclement can no longer be considered adiabatic [139]. Such dynamical evolutions are known to give rise to chiral mode conversions, where the final state of the system is determined by the direction of EP encirclement [140]. This exotic behavior has been experimentally 
demonstrated in a coupled optical waveguide arrangement [141]. By properly designing the boundaries and losses of each waveguide, a dynamical EP encirclement could be effectively realized in the parameter domain. Owing to the chiral behavior, the output of the system toggles between the even and odd supermodes depending on the direction of propagation of light, regardless of the input beams. A parallel experiment on this chiral mode conversion was carried out in an optomechanical system, where a nonreciprocal transfer was observed between two vibrational modes of a silicon membrane embedded in a high-finesse optical cavity [142]. Similar effects have also been observed in silicon-based photonic architectures [143]. More recently, an analytical explanation of this chiral and robust state conversion mechanism was provided through the asymptotics of exact solutions, along with the fact that this effect can persist even in the presence of nonlinearities [144, 145]. In addition, the possibility of a single-channel optical omni-polarizer was proposed that benefits from this chiral response [144]. Finally, some of the peculiar features arising from the process of winding around multiple $\mathrm{NH}$ singularities or
EPs have also been explored by invoking the topological notion of homotopy (Figure 4A) [146].

\subsection{Symmetries and topology meet EPs}

The recent advances in the field of $\mathrm{NH}$ physics has incited a flurry of research activities aimed at understanding the interplay between symmetries and topology on the one hand and EPs on the other hand. Such studies can provide a guideline to achieving new symmetry-protected $\mathrm{NH}$ phases that have no counterpart whatsoever in the Hermitian domain. For instance, it has been shown that a Dirac point with a nontrivial Berry phase can split into isolated pairs of EPs in the presence of non-Hermiticity [147]. The ensuing double Riemann sheet associated with these EP pairs in turn leads to a bulk Fermi arc which bridges the two EPs in the complex band structure (Figure 4B). This latter effect is a direct by-product of non-Hermiticity and is different from surface Fermi arcs that arise from Weyl points in 3D Hermitian systems. In addition, the EPs obtained in this fashion exhibit half-integer topological
A

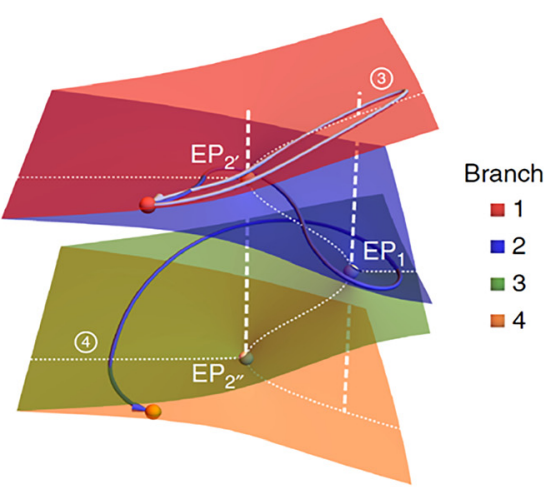

B

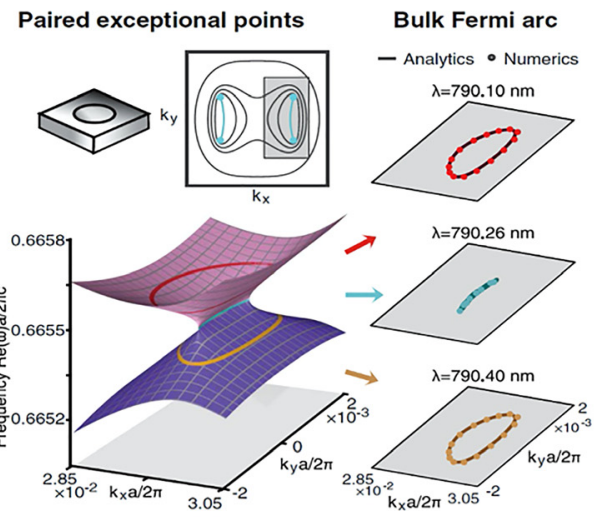

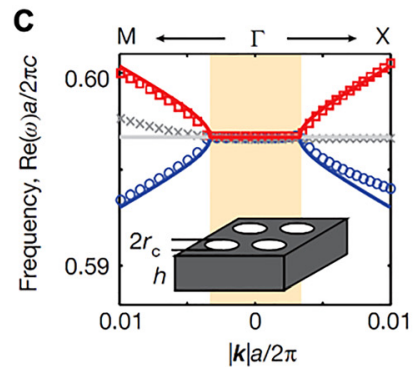

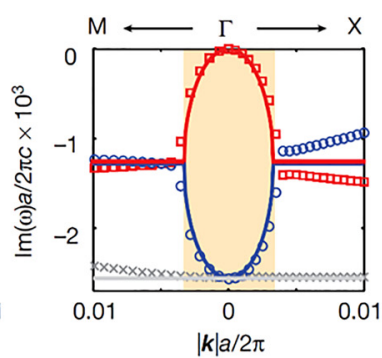

D
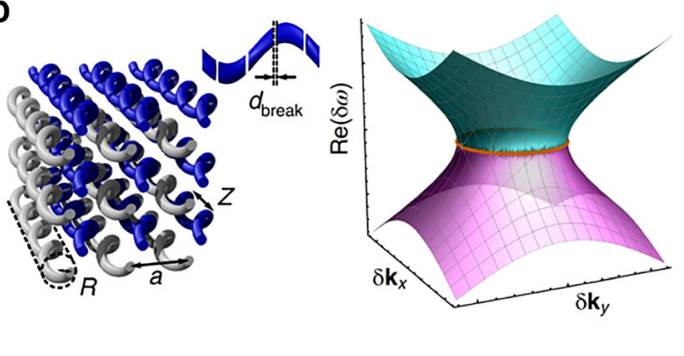

Figure 4: Exceptional rings and higher-order effects.

(A) Exceptional point (EP) encirclement in the parameter space of a system supporting two EPs. In this case, nonhomotopic loops encircling EP1 indicated in the diagram result in different dynamic and stroboscopic evolution behaviors [146]. (B) A bulk Fermi arc that connects a pair of EPs arising from a single Dirac point in the presence of radiation losses in a rhombic lattice having elliptical air holes embedded in a dielectric substrate [147]. (C) Real and imaginary parts of the complex band structure associated with a non-Hermitian square lattice photonic crystal exhibiting a ring of EPs [150]. (D) Helical waveguide lattices with controllable losses utilized to observe a Weyl exceptional ring in their corresponding band structure [156]. 
invariants, which could manifest themselves in the farfield polarization of the light scattered by the implemented photonic crystal [147]. Along similar lines, there have been proposals to observe bulk Fermi arcs in solid state, where the required non-Hermiticity could be induced via ferromagnetic leads attached to a TI [148].

A Dirac Hamiltonian that deviates from the Hermitian regime is also known to exhibit other interesting effects. To this end, a circuit realization of NH Dirac and Weyl Hamiltonians under the influence of a pseudomagnetic field which is artificially induced by a judicious spatial variation of the circuit elements has been proposed [149]. In this scenario, the combined effect of non-Hermiticity along with the pseudomagnetic field lifts the degeneracy and leads to the emergence of Landau-level-like flat bands in the band structure of the system. Another example involves an accidental degeneracy in the form of a Dirac cone in the Hermitian band structure of a square-lattice photonic crystal [150]. Once non-Hermiticity is at play in the form of radiation losses, this Dirac point transforms into a ring of EPs (Figure 4C) which manifest themselves in the angleresolved reflection measurements.

Another topic of interest is the synergy between symmetry and EPs. In this vein, symmetry-protected exceptional surfaces are known to generically arise in $\mathrm{NH}$ band structures with an increased dimensionality as compared with the case with no symmetries [151]. In this context, $\mathrm{NH}$ symmetries can transport nodal $\mathrm{NH}$ semimetals into symmetry-protected $\mathrm{NH}$ metals. Other works have also investigated these concepts in nonconservative systems, where exceptional surfaces are protected by various constraints such as PT and parity-particle-hole (CP) symmetries [128, 152]. Similar studies have predicted symmetryprotected exceptional rings which are characterized by nonzero topological invariants [153].

In Hermitian topological physics, an important family of degeneracies are the celebrated Weyl points. Such degeneracy points can be interpreted as magnetic monopoles in reciprocal space which are characterized by a quantized Chern number [154]. A closely related concept is a Weyl nodal ring, which is essentially a 3D generalization of Dirac nodes. These latter rings happen to have trivial Chern invariants while acquiring a nonzero Berry phase over a closed path that encircles the entire ring in the momentum space. Quite recently, a set of $\mathrm{NH}$ degeneracies termed Weyl exceptional rings have been theoretically predicted which feature both a nontrivial Chern number and a quantized nonzero Berry phase [155]. This intriguing behavior stems from the topology of the Riemann surface which is unique to $\mathrm{NH}$ arrangements. Weyl exceptional rings were later experimentally demonstrated in a Floquet system realized in a 3D photonic bipartite lattice comprising evanescently coupled helical waveguides written in silica (Figure 4D) [156]. The required nonHermiticity in this platform is obtained by incorporating equidistant breaks within the waveguides in one of the sublattices to impose a controlled amount of loss. Experimental measurements confirm a topological transition where Fermi arc surface states emerge in the system after increasing the dissipation levels.

\section{Non-Hermitian topological physics}

Topological physics is an emerging field that aims to understand and harness a set of new properties arising in a recently discovered phase of matter - properties that tend to remain invariant during a continuous deformation of the system [21-23]. A prime example of such material systems is that of TIs, where electron conduction occurs along the edges while it is prohibited in the bulk [157-160]. In recent years, the prospect of using topological notions in optics to utilize the unique attributes offered by topologically nontrivial structures has been the subject of intense research efforts [25, 26, 161-164]. In this regard, unidirectional transport and robust topological edge modes have been demonstrated in coupled resonators and waveguide lattices [24-26]. The field of topological photonics took a drastic turn after the pioneering experiment of Ref. [25] that demonstrated topologically protected light transport in a magnet-free photonic structure. Quite recently, the field also made a substantial leap forward after optical amplification/attenuation was introduced in conjunction with topology. In this regard, it has been shown that the synergy between non-Hermiticity and topology can lead to more efficient coherent light sources with superior performance in terms of robustness and emission characteristics. In addition, the very possibility of realizing $\mathrm{NH}$ topological systems in photonic platforms has led to a new field of research, namely $\mathrm{NH}$ topological physics, which makes an effort to understand and predict the response of topological phases in the presence of non-Hermiticity $[165,166]$. In this section, we summarize some of the advances in this exciting field.

\subsection{Topological lasers}

The fact that lasers are susceptible to defects and disorder has always posed a significant challenge in the 
performance of these devices. Such imperfections are in general inevitable during the fabrication process or may develop in time due to operational degradation and malfunction. These in turn could lead to spatial localization of light within the cavity, eventually leading to lower output powers or even a sudden shutdown of the laser itself. In addition, on many occasions, an array of coupled lasers is used to boost the total emitted power via coherent constructive interference among individual elements. In such scenarios, the system would be even more prone to random deficiencies and failure. It is in this vein that topological features have been sought as a means to develop laser systems that could be immune to perturbations.

In one dimension, an archetypical topological structure is that offered by the Su-Schrieffer-Heeger (SSH) model [167]. When terminated properly, such an array hosts topologically protected defect states with eigenvalues that reside in the middle of the bandgap and are robust against structural disorders. Lasing in this defect state has been demonstrated in systems involving polaritons (Figure 5A) [168], microring cavities (Figure 5B, C) $[169,170]$, and photonic crystals [171]. Depending on the pumping pattern used, this $\mathrm{NH}$ lattice exhibits different regimes of behavior, as dictated by its associated symmetries $[169,172]$. In this regard, by appropriately distributing optical gain and loss among the constituent elements, the defect state can be induced to lase in a single-mode fashion while maintaining its topological features in the $\mathrm{NH}$ domain. In particular, the lasing edge mode in the $\mathrm{SSH}$ array was found to be resilient to both on-site and tunneling disorders $[168,170]$. Moreover, unlike the bulk modes, this edge state lases at a wavelength that tends to remain unperturbed even at high pump power levels where non-Hermiticity plays an important role [169].
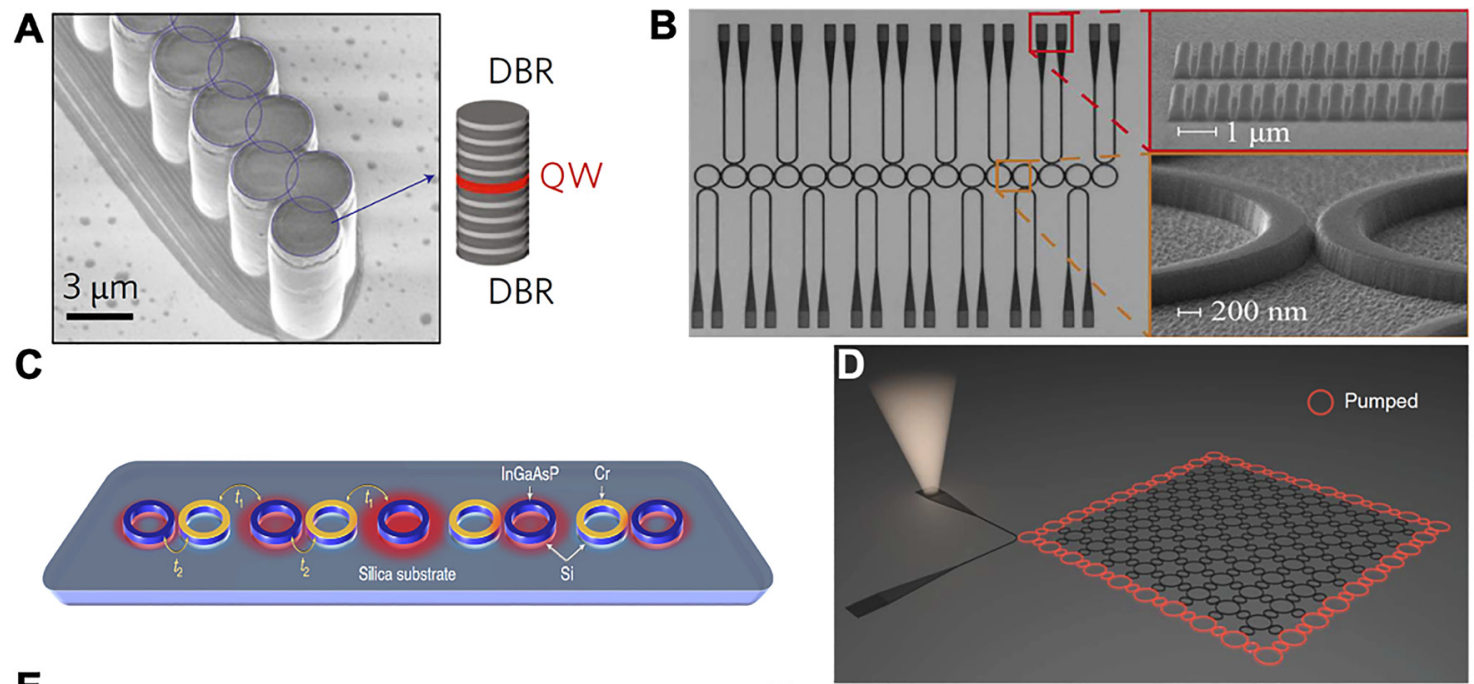

$\mathbf{E}$

$\mathbf{F}$
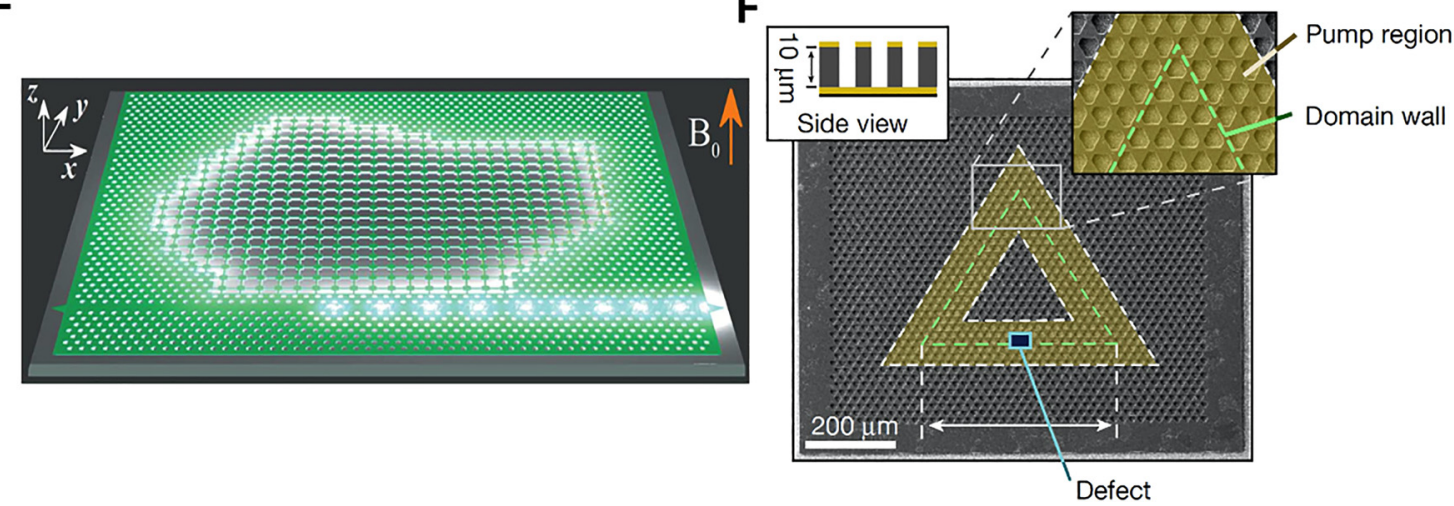

Figure 5: Topological lasers.

(A) Lasing in the topological defect state of a one-dimensional Su-Schrieffer-Heeger (SSH) array of polaritonic micropillar cavities [168]. (B, C) Similar SSH structures implemented based on microring resonators [169, 170]. (D) All-dielectric two-dimensional (2D) topological laser array using microrings coupled via intermediary links to induce an artificial gauge field [174]. (E) Topological lasing in a photonic crystal fabricated on an yttrium iron garnet (YIG) substrate [175]. (F) Electrically pumped topological laser demonstrated in a valley photonic crystal based on quantum cascade lasers (QCLs) [178]. 
The concept of topological edge transport in a twodimensional (2D) laser array is yet another avenue that has been pursued in a number of platforms. In this respect, various methods have been used to induce a topologically nontrivial response in these active settings. The prospect of realizing 2D topological lasers has also been pursued in alldielectric photonic platforms. To achieve this, arrays of coupled microcavities with asymmetric intermediary rings have been used to emulate the presence of an artificial magnetic field (see Figure 5D) [173, 174]. By pumping the boundaries of such a lattice, topological transport in the lasing edge mode has been reported. As compared with a similar but trivial array, higher slope efficiencies and robust single-mode operation is observed even for pump intensities high above threshold. Along different lines, a topologically nontrivial square lattice has been implemented by depositing magneto-optic materials in the substrate of a photonic crystal, where time-reversal symmetry (TRS) can be broken when applying a magnetic field (Figure 5E) [175]. The resulting topological structure is then optically pumped, thus promoting unidirectional lasing along the interface of this square lattice with its surrounding topologically trivial triangular crystal. Similar schemes have been utilized in exciton-polaritonic systems [176]. Alternatively, topological features can be induced in a crystal via the valley degree of freedom [177]. Using this technique, electrically pumped topological lasers have been experimentally realized in the $\mathrm{THz}$ regime in a valley photonic crystal inscribed in a QCL wafer (Figure 5F) [178, 179]. The lasing edge mode in such a device can be immune to backscattering due to defects that do not cause intervalley scattering. Interestingly, the role of topology in lasers is not limited to the edge states confined at the boundaries of a photonic system. Rather, it has been suggested that by judiciously interfacing topological and trivial crystals, one can form a highly confined 2D cavity enabled by band inversion reflections [180]. This reflection mechanism can then lead to single-mode lasing with high vertical directionality.

\subsection{Non-Hermitian symmetries and topology}

In the Hermitian domain, it is well known that symmetries play a pivotal role in topological arrangements. In 2D systems, for example, a Chern insulator could be obtained when the TRS is broken [181]. In fact, the concept of topological protection is often closely intertwined with certain types of symmetries associated with the system [22, 158]. When generalizing topological notions to $\mathrm{NH}$ systems, it is therefore natural to ask as to how NH symmetries would interact with topology. Do topologically nontrivial phases exist in certain open systems in various dimensions that satisfy a specific type of symmetry? Can NH symmetries protect a topological edge state?

Answering some of the aforementioned questions has been the subject of numerous recent studies. The existence of topologically protected defect states in PT-symmetric $1 \mathrm{D}$ SSH lattices has been predicted and observed in passive coupled waveguides inscribed in silica (Figure 6A) [182, 183]. Various regimes of PT-broken and unbroken were examined as a function of the dimerization in this same structure, and the onset of topological phase transition was shown to be linked to the mean displacement of the light traveling in this discrete lattice. The role of $\mathrm{NH}$ symmetries in protecting the defect state of an SSH topological array that respects PT symmetry has also been studied [184]. In such cases, unlike the trivial eigenstates, the topological defect modes preserve their associated eigenvalues even in the presence of perturbations that respect PT symmetry. Remarkably, in some cases, $\mathrm{NH}$ symmetries like for example that of $\mathrm{NH}$ charge-conjugation or PT symmetry can be the origin of topological edge states, even when a similar Hermitian system is topologically trivial [172, 185, 186]. Therefore, the corresponding defect state resides at the boundary of two regions that are characterized by different $\mathrm{NH}$ parameters, and is known to emerge from a continuum in the band structure. Similar effects have been studied in a PT-symmetric Aubry-André-Harper (AAH) model [187].

As mentioned earlier, breaking TRS is yet another way to endow a physical system with nontrivial topological properties. In static optical systems, however, such TRS breaking can typically emerge from gyromagnetic effects [24, 161, 162]. In this regard, alternative methodologies geared at breaking TRS in the NH domain have been pursued, like for example, Floquet systems in 1D quantum walks [188] and 2D lattices for implementing Chern insulators $[189,190]$. Alternative techniques for realizing Chern insulators in active platforms have also been suggested that rely on the interplay between nonlinearity and non-Hermiticity [191]. On a different front, endowing topological systems with $\mathrm{NH}$ symmetries has been used to develop optical devices with new functionalities. These include PT-symmetric resonator arrays in microwave systems (see Figure 6B) [192], optical isolators in waveguide arrays [193], optical limiters [194], and microring laser arrays capable of light steering [195].

There are currently ongoing efforts aiming to find a unified classification of topological $\mathrm{NH}$ systems with different types of symmetries [196, 197]. In this respect, 
A

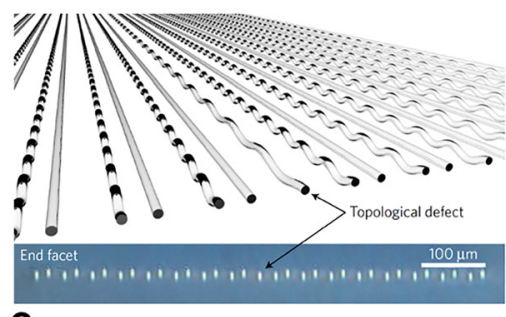

C

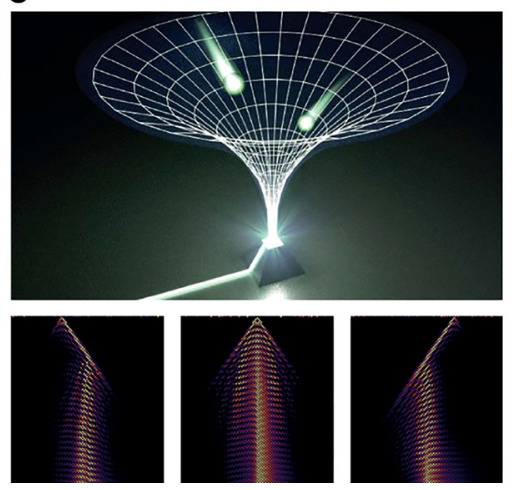

B

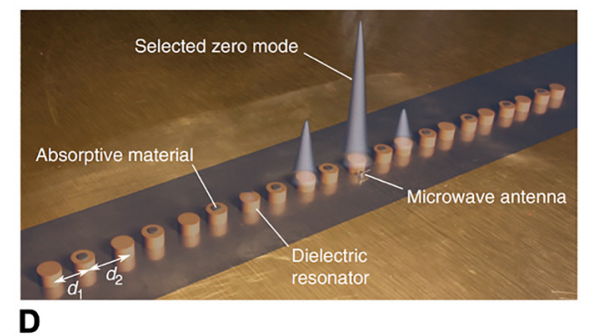

D

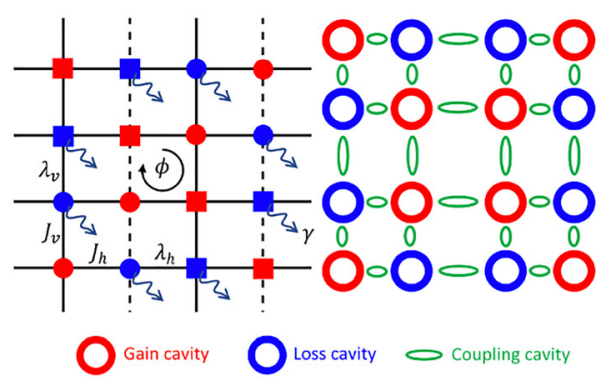

Figure 6: Symmetries and higher-order effects in non-Hermitian $(\mathrm{NH})$ topological systems.

(A) Parity-time (PT)-symmetric SuSchrieffer-Heeger (SSH) Hamiltonian realized in a waveguide array inscribed in fused silica [182]. (B) Selective enhancement of a topological defect state in a one-dimensional (1D) array of dielectric microwave resonators by utilizing PT-symmetry [192]. (C) Topological funneling of light via the $\mathrm{NH}$ skin effect in a 1D chain with anisotropic couplings (top). The input beam is always funneled through the topological interface localized in the middle of the structure, irrespective of its initial launching position (bottom) [209]. (D) A two-dimensional (2D) NH model which supports second-order topological corner states (left). Such a system could be physically realized using microring resonators with intermediary links (right) [219]. even though similar studies in Hermitian TIs $[198,199]$ can provide a baseline, studies suggest stark distinctions between such arrangements and their $\mathrm{NH}$ analogs. For instance, it has been found that the $\mathrm{NH}$ counterparts of some of the fundamental symmetries, which are distinct in the Hermitian regime, are in fact equivalent to each other and can be unified [200]. This could lead to nonequilibrium states that are unique to $\mathrm{NH}$ topological settings, and implies that in general, the topological classifications of $\mathrm{NH}$ symmetries are expected to be quite different from the ones known in the Hermitian domain.

\subsection{Non-Hermitian bulk-edge correspondence and non-Hermitian skin effects}

The hallmark of topological phases is the presence of edge states that emerge at the boundaries between structures with different topological invariants. In the Hermitian regime, this exotic behavior is a by-product of the bulk-boundary correspondence which relates the topological properties of bulk media to their boundary states [22]. Nevertheless, despite a growing interest in $\mathrm{NH}$ topological systems and their applications [46], it is not immediately clear how this correspondence could be translated to such settings [201-204]. Perhaps, a prominent example of how conventional bulkboundary correspondence could no longer hold in the $\mathrm{NH}$ regime is the so-called "non-Hermitian skin effect" [202, 205208]. In accordance with this, an NH structure with extended bulk states under periodic boundary conditions could behave in a completely different way when terminated with open boundaries. In particular, for certain regions of the $\mathrm{NH}$ parameter space, such bulk modes all collapse into localized edge modes - a clear violation of the standard bulkboundary correspondence. This interesting $\mathrm{NH}$ phenomenon has recently been observed in photonic mesh lattices (Figure 6C) [209] and is currently the subject of further studies that aim to shed light on how different NH symmetries could modify the skin effect [196, 207].

Attempts to explain the NH skin effect have been at the core of developing an appropriately modified formalism that could successfully describe the bulk-boundary correspondence in the $\mathrm{NH}$ domain. In this regard, two main methods have been implemented so far, each focusing on either the complex spectra of the corresponding $\mathrm{NH}$ Hamiltonian and its associated point/line degeneracies $[196,207,210]$, or the non-Bloch nature of the eigenstates in an open boundary geometry [205, 206, 211]. In the first approach, topological invariants are interpreted in terms of dynamical phases which depend not only on the eigenstates of the Hamiltonian, but also the associated complex eigenspectrum. In this context, similar to the concept of a topological bandgap in the Hermitian domain, an $\mathrm{NH}$ system can be considered as topologically nontrivial if its complex energy spectrum encircles a prespecified base point in the complex plane [210]. Using this, a new kind of bulk-boundary correspondence is then established, wherein a winding number is defined as the degeneracy at the chosen base point. This latter topological invariant 
represents the number of independent edge states in a semi-infinite arrangement. In this formalism, one finds that unlike Hermitian topological lattices which require at least two bands in their band structure, an NH system could exhibit topological behavior in the absence of any symmetry constraints even within a single band. Other results also related to the role of $\mathrm{NH}$ degeneracies in this matter have been reported [212].

Alternatively, the breakdown of the conventional bulkboundary correspondence in $\mathrm{NH}$ arrangements can be attributed to the non-Bloch nature of the eigenstates in such systems. To address this issue, a non-Bloch Chern number could be defined in a 2D lattice which successfully takes into account the aforementioned NH skin effect [206]. Considering a modified $\mathrm{NH}$ version of the Haldane model, it has been shown that while the Hermitian Chern number fails to capture topological phase transitions, the new $\mathrm{NH}$ Chern number successfully predicts such topological phases and is a faithful indicator of the number of chiral edge modes in this scenario. In contrast to the first approach mentioned previously, this formalism explicitly relies on the non-Bloch eigenstates to redefine the bulkedge correspondence. Similar findings have been also presented in 1D NH settings [205, 211].

We would like to mention that there are several modifications of the non-Bloch formalism that intend to introduce a self-consistent framework for $\mathrm{NH}$ topological systems. One such result exploits the biorthogonal basis for $\mathrm{NH}$ Hamiltonians to redefine the bulk as well as edge states in terms of the left and right eigenstates [213]. Based on these, a biorthogonal polarization parameter is then introduced that is shown to be quantized and capable of describing the topological properties of the corresponding $\mathrm{NH}$ structure. In addition, it has been suggested that by defining a modified version of the periodic boundary conditions, one could restore the Hermitian bulk-boundary correspondence even in the presence of non-Hermiticity [214]. This could be achieved by introducing a new parameter in this type of boundary conditions. The resulting generalized parameter space can then be used to bridge the open boundary skin effects with the bulk Hamiltonian in a periodic geometry.

\subsection{Higher-order non-Hermitian topological effects}

In a conventional $N$-dimensional TI, a nontrivial topological invariant guarantees the existence of $\mathrm{N}$-1-dimensional gapless edge states. Examples include topological transport of surface states and edge modes in 3D and 2D systems, respectively. This paradigm was extended recently [215] by introducing higher-order topological insulators (HOTI), wherein generalized multipole moments are posed as quantized electromagnetic observables [215217]. By using Wilson loop operators, these quantized multipole moments are proved to act as topological invariants that can lead to topologically protected boundary states. Examples of such HOTIs include second-order 2D and 3D TIs with topologically protected corner and hinge states, respectively [217].

In the context of $\mathrm{NH}$ systems, higher-order topological effects could lead to interesting effects that are absent in the Hermitian domain [218]. For instance, HOTIs could arise as a result of non-Hermiticity [219]. This can happen for example in a 2D array of coupled microring cavities with on-site gain and loss arranged in a staggered manner (Figure 6D). In this scenario, although the structure is topologically trivial in the Hermitian limit, by increasing the gain/loss levels above a certain threshold value, a higher-order topological phase transition occurs. The resulting second-order TI is a host to four degenerate corner states in the boundaries of the structure. This behavior can be justified by using a biorthogonal form of nested Wilson loops to establish a higherorder bulk-boundary correspondence [219]. Along different lines, higher-order corner modes within the skin states of $\mathrm{NH}$ 2D and 3D lattices have been studied [220] both in reciprocal and nonreciprocal regimes. These $\mathrm{NH}$ behaviors have been shown to be governed by a biorthogonal bulk-boundary correspondence [213].

As mentioned before, the usual bulk-boundary correspondence defined for Hermitian TIs can break down in the presence of non-Hermiticity. Interestingly, the higherorder bulk-boundary correspondence could also be modified when translated to the $\mathrm{NH}$ domain [221]. In this context, symmetry protected second-order TIs with corner states localized asymmetrically in one boundary have been predicted in 2D NH lattices [221, 222]. In 3D, the breakdown of Hermitian bulk-boundary correspondence can lead to the emergence of anomalous second-order corner modes instead of hinge states. This discrepancy can be rectified via proper use of a non-Bloch eigenstate formalism [221].

\section{Summary}

In this article, we provided an overview of the recent advances in the field of $\mathrm{NH}$ optics. We discussed how various optical platforms exhibiting gain and loss can be utilized to investigate different aspects associated with PT symmetry and $\mathrm{NH}$ phenomena. In addition to enabling new functionalities within the discipline of photonics that have no 
counterparts in the Hermitian domain, such optical realizations have also played a key role in the emerging field of $\mathrm{NH}$ topological physics that aims to understand and utilize the synergy between topological notions and nonHermiticity. More importantly, although PT symmetry, EPs, and the interplay of topology and non-Hermiticity have been extensively explored in the classical and semiclassical regimes, still little is known about the ramification of these developments in a fully quantum domain. In addition, despite intensive recent efforts, a universal theory of $\mathrm{NH}$ bulk-boundary correspondence that selfconsistently describes topologically nontrivial behavior in all dimensionalities is still elusive. Given that many of these aspects remain unexplored, we believe that further activities in this field will not only yield results that are fundamental in nature, but they could also introduce new tools in photonics and other fields for a new generation of devices and systems.

Acknowledgments: The authors gratefully acknowledge the financial support from DARPA (D18AP00058, HR00111820042, HR00111820038), Army Research Office (ARO; W911NF-16-1-0013, W911NF-17-1-0481), National Science Foundation (ECCS 1454531, DMR 1420620, ECCS 1757025, CBET 1805200, ECCS 2000538, ECCS 2011171), Office of Naval Research (N0001416-1-2640, N00014-18-12347, N00014-19-1-2052), Air Force Office of Scientific Research (FA9550-14-1-0037) and US-Israel Binational Science Foundation (BSF; 2016381).

Author contribution: All the authors have accepted responsibility for the entire content of this submitted manuscript and approved submission.

Research funding: We gratefully acknowledge the financial support from DARPA (D18AP00058, HR00111820042, HR00111820038), Army Research Office (ARO; W911NF-16-1-0013, W911NF-17-1-0481), National Science Foundation (ECCS 1454531, DMR 1420620, ECCS 1757025, CBET 1805200, ECCS 2000538, ECCS 2011171), Office of Naval Research (N0001416-1-2640, N00014-18-12347, N00014-19-1-2052), Air Force Office of Scientific Research (FA9550-14-1-0037) and US-Israel Binational Science Foundation (BSF; 2016381).

Conflict of interest statement: The authors declare no conflicts of interest regarding this article.

\section{References}

[1] G. Gamow, "Zur Quantentheorie des Atomkernes," Z. Phys., vol. 51, pp. 204-212, 1928.
[2] H. Feshbach, C. E. Porter, and V. F. Weisskopf, "Model for nuclear reactions with neutrons," Phys. Rev., vol. 96, pp. 448-464, 1954.

[3] C. M. Bender and S. Boettcher, "Real spectra in non-Hermitian Hamiltonians having PT symmetry,” Phys. Rev. Lett., vol. 80, pp. 5243-5246, 1998.

[4] C. M. Bender, S. Boettcher, and P. N. Meisinger, "PT-symmetric quantum mechanics,” J. Math. Phys., vol. 40, pp. 2201-2229, 1999.

[5] R. El-Ganainy, K. G. Makris, D. N. Christodoulides, and Z. H. Musslimani, "Theory of coupled optical PT-symmetric structures,” Opt. Lett., vol. 32, pp. 2632-2634, 2007.

[6] K. G. Makris, R. El-Ganainy, D. N. Christodoulides, and Z. H. Musslimani, "Beam dynamics in PT symmetric optical lattices," Phys. Rev. Lett., vol. 100, p. 103904, 2008.

[7] Z. H. Musslimani, K. G. Makris, R. El-Ganainy, and D. N. Christodoulides, "Optical solitons in PT periodic potentials,” Phys. Rev. Lett., vol. 100, p. 030402, 2008.

[8] A. Guo, G. J. Salamo, D. Duchesne, et al., "Observation of PT-symmetry breaking in complex optical potentials," Phys. Rev. Lett., vol. 103, p. 093902, 2009.

[9] C. E. Rüter, K. G. Makris, R. El-Ganainy, D. N. Christodoulides, M. Segev, and D. Kip, "Observation of parity-time symmetry in optics,” Nat. Phys., vol. 6, pp. 192-195, 2010.

[10] S. Klaiman, U. Günther, and N. Moiseyev, "Visualization of branch points in PT-symmetric waveguides," Phys. Rev. Lett., vol. 101, p. 080402, 2008.

[11] S. Bittner, B. Dietz, U. Günther, et al., "PT symmetry and spontaneous symmetry breaking in a microwave billiard," Phys. Rev. Lett., vol. 108, p. 024101, 2012.

[12] J. Schindler, A. Li, M. C. Zheng, F. M. Ellis, and T. Kottos, "Experimental study of active LRC circuits with PT symmetries," Phys. Rev. A, vol. 84, p. 040101, 2011.

[13] C. M. Bender, B. K. Berntson, D. Parker, and E. Samuel, "Observation of PT phase transition in a simple mechanical system,” Am. J. Phys., vol. 81, pp. 173-179, 2013.

[14] H. Jing, S. K. Özdemir, X.-Y. Lü, J. Zhang, L. Yang, and F. Nori, “PT-symmetric phonon laser," Phys. Rev. Lett., vol. 113, p. $053604,2014$.

[15] H. Jing, Ş. K. Özdemir, Z. Geng, et al., “Optomechanicallyinduced transparency in parity-time-symmetric microresonators," Sci. Rep., vol. 5, p. 9663, 2015.

[16] X. Zhu, H. Ramezani, C. Shi, J. Zhu, and X. Zhang, "PT-symmetric acoustics,” Phys. Rev. X, vol. 4, p. 031042, 2014.

[17] R. Fleury, D. Sounas, and A. Alù, "An invisible acoustic sensor based on parity-time symmetry," Nat. Commun., vol. 6, p. 5905, 2015.

[18] C. Hang, G. Huang, and V. V. Konotop, "PT symmetry with a system of three-level atoms," Phys. Rev. Lett., vol. 110, p. 083604, 2013

[19] Z. Zhang, Y. Zhang, J. Sheng, et al., "Observation of parity-time symmetry in optically induced atomic lattices," Phys. Rev. Lett., vol. 117, p. 123601, 2016.

[20] P. Peng, W. Cao, C. Shen, et al., "Anti-parity-time symmetry with flying atoms," Nat. Phys., vol. 12, pp. 1139-1145, 2016.

[21] D. J. Thouless, M. Kohmoto, M. P. Nightingale, and M. den Nijs, "Quantized Hall conductance in a two-dimensional periodic potential," Phys. Rev. Lett., vol. 49, pp. 405-408, 1982.

[22] M. Z. Hasan and C. L. Kane, "Colloquium: topological insulators,” Rev. Mod. Phys., vol. 82, pp. 3045-3067, 2010. 
[23] X.-L. Qi and S.-C. Zhang, "Topological insulators and superconductors," Rev. Mod. Phys., vol. 83, pp. 1057-1110, 2011.

[24] Z. Wang, Y. D. Chong, J. D. Joannopoulos, and M. Soljačić, "Reflection-free one-way edge modes in a gyromagnetic photonic crystal," Phys. Rev. Lett., vol. 100, p. 013905, 2008.

[25] M. C. Rechtsman, J. M. Zeuner, Y. Plotnik, et al., "Photonic Floquet topological insulators," Nature, vol. 496, pp. 196-200, 2013.

[26] M. Hafezi, S. Mittal, J. Fan, A. Migdall, and J. M. Taylor, "Imaging topological edge states in silicon photonics," Nat. Photonics, vol. 7, pp. 1001-1005, 2013.

[27] E. Yablonovitch, "Inhibited spontaneous emission in solid-state physics and electronics," Phys. Rev. Lett., vol. 58, pp. 2059-2062, 1987.

[28] S. John, "Strong localization of photons in certain disordered dielectric superlattices," Phys. Rev. Lett., vol. 58, pp. 2486-2489, 1987.

[29] J. C. Knight, J. Broeng, T. A. Birks, and P. S. J. Russell, "Photonic band gap guidance in optical fibers," Science, vol. 282, pp. 1476-1478, 1998.

[30] V. M. Shalaev, “Optical negative-index metamaterials," Nat. Photonics, vol. 1, pp. 41-48, 2007.

[31] I. Vorobeichik, U. Peskin, and N. Moiseyev, "Modal losses and design of modal irradiance patterns in an optical fiber by the complex scaled (t, t') method," J. Opt. Soc. Am. B, vol. 12, pp. 1133-1141, 1995.

[32] I. Vorobeichik, N. Moiseyev, D. Neuhauser, M. Orenstein, and U. Peskin, "Calculation of light distribution in optical devices by a global solution of an inhomogeneous scalar wave equation," IEEE J. Quant. Electron., vol. 33, pp. 1236-1244, 1997.

[33] I. Vorobeichik, N. Moiseyev, and D. Neuhauser, "Effect of the second-derivative paraxial term in the scalar Maxwell's equation on amplitude losses and reflections in optical fibers,"J. Opt. Soc. Am. B, vol. 14, pp. 1207-1212, 1997.

[34] A. M. Kennis, I. Vorobeichik, and N. Moiseyev, "Analysis of an intermediate-mode-assisted directional coupler using Bloch theory," IEEE J. Quant. Electron., vol. 36, pp. 563-573, 2000.

[35] K. Gokhberg, I. Vorobeichik, E. Narevicius, and N. Moiseyev, "Solution of the vector wave equation by the separable effective adiabatic basis set method," J. Opt. Soc. Am. B, vol. 21, pp. 1809-1817, 2004.

[36] B. Alfassi, O. Peleg, N. Moiseyev, and M. Segev, "Diverging Rabi oscillations in subwavelength photonic lattices," Phys. Rev. Lett., vol. 106, p. 073901, 2011.

[37] A. Pick and N. Moiseyev, "Polarization dependence of the propagation constant of leaky guided modes," Phys. Rev. A, vol. 97, p. 043854, 2018.

[38] N. Moiseyev, L. D. Carr, B. A. Malomed, and Y. B. Band, "Transition from resonances to bound states in nonlinear systems: application to Bose-Einstein condensates," J. Phys. B: At. Mol. Opt. Phys., vol. 37, pp. L193-L200, 2004.

[39] N. Moiseyev and L. S. Cederbaum, "Resonance solutions of the nonlinear Schrödinger equation: tunneling lifetime and fragmentation of trapped condensates," Phys. Rev. A, vol. 72, p. 033605, 2005.

[40] O. Peleg, M. Segev, G. Bartal, D. N. Christodoulides, and N. Moiseyev, "Nonlinear waves in subwavelength waveguide arrays: evanescent bands and the "Phoenix Soliton", Phys. Rev. Lett., vol. 102, p. 163902, 2009.
[41] O. Peleg, Y. Plotnik, N. Moiseyev, O. Cohen, and M. Segev, "Selftrapped leaky waves and their interactions," Phys. Rev. A, vol. 80, p. 041801, 2009.

[42] K. Petermann, "Calculated spontaneous emission factor for double-heterostructure injection lasers with gain-induced waveguiding," IEEE J. Quant. Electron., vol. 15, pp. 566-570, 1979.

[43] A. E. Siegman, "Excess spontaneous emission in non-Hermitian optical systems. I. Laser amplifiers," Phys. Rev. A, vol. 39, pp. 1253-1263, 1989.

[44] W. A. Hamel and J. P. Woerdman, "Observation of enhanced fundamental linewidth of a laser due to nonorthogonality of its longitudinal eigenmodes," Phys. Rev. Lett., vol. 64, pp. 1506-1509, 1990.

[45] A. L. Schawlow and C. H. Townes, "Infrared and optical masers," Phys. Rev., vol. 112, pp. 1940-1949, 1958.

[46] G. Yoo, H.-S. Sim, and H. Schomerus, "Quantum noise and mode nonorthogonality in non-Hermitian PT-symmetric optical resonators," Phys. Rev. A, vol. 84, p. 063833, 2011.

[47] B. Peng, Ş. K. Özdemir, F. Lei, et al., "Parity-time-symmetric whispering-gallery microcavities," Nat. Phys., vol. 10, pp. 394-398, 2014.

[48] L. Chang, X. Jiang, S. Hua, et al., "Parity-time symmetry and variable optical isolation in active-passive-coupled microresonators," Nat. Photonics, vol. 8, pp. 524-529, 2014.

[49] M. Liertzer, L. Ge, A. Cerjan, A. D. Stone, H. E. Türeci, and S. Rotter, "Pump-induced exceptional points in lasers," Phys. Rev. Lett., vol. 108, p. 173901, 2012.

[50] M. Brandstetter, M. Liertzer, C. Deutsch, et al., "Reversing the pump dependence of a laser at an exceptional point," Nat. Commun., vol. 5, p. 4034, 2014.

[51] B. Peng, Ş. K. Özdemir, S. Rotter, et al., “Loss-induced suppression and revival of lasing," Science, vol. 346, pp. 328-332, 2014.

[52] B. Peng, Ş. K. Özdemir, M. Liertzer, et al., "Chiral modes and directional lasing at exceptional points," Proc. Natl. Acad. Sci. U. S. A., vol. 113, pp. 6845-6850, 2016.

[53] J. Zhang, B. Peng, Ş. K. Özdemir, et al., "A phonon laser operating at an exceptional point," Nat. Photonics, vol. 12, pp. 479-484, 2018.

[54] M.-A. Miri, P. LiKamWa, and D. N. Christodoulides, "Large area single-mode parity-time-symmetric laser amplifiers," Opt. Lett., vol. 37, p. 764, 2012.

[55] H. Hodaei, M.-A. Miri, M. Heinrich, D. N. Christodoulides, and M. Khajavikhan, "Parity-time-symmetric microring lasers," Science, vol. 346, pp. 975-978, 2014.

[56] L. Feng, Z. J. Wong, R.-M. Ma, Y. Wang, and X. Zhang, "Singlemode laser by parity-time symmetry breaking," Science, vol. 346, pp. 972-975, 2014.

[57] H. Hodaei, M.-A. Miri, A. U. Hassan, et al., "Single mode lasing in transversely multi-moded PT-symmetric microring resonators," Laser Photonics Rev., vol. 10, pp. 494-499, 2016.

[58] W. Liu, M. Li, R. S. Guzzon, et al., "An integrated parity-time symmetric wavelength-tunable single-mode microring laser," Nat. Commun., vol. 8, p. 15389, 2017.

[59] W. E. Hayenga, H. Garcia-Gracia, E. Sanchez Cristobal, et al., "Electrically pumped microring parity-time-symmetric lasers," Proc. IEEE, vol. 108, pp. 827-836, 2020. 
[60] A. K. Jahromi, A. U. Hassan, D. N. Christodoulides, and A. F. Abouraddy, "Statistical parity-time-symmetric lasing in an optical fibre network," Nat. Commun., vol. 8, p. 1359, 2017.

[61] C. M. Gentry and M. A. Popović, "Dark state lasers," Opt. Lett., vol. 39, pp. 4136-4139, 2014.

[62] H. Hodaei, A. U. Hassan, W. E. Hayenga, M. A. Miri, D. N. Christodoulides, and M. Khajavikhan, "Dark-state lasers: mode management using exceptional points," Opt. Lett., vol. 41, pp. 3049-3052, 2016.

[63] A. Marandi, Z. Wang, K. Takata, R. L. Byer, and Y. Yamamoto, "Network of time-multiplexed optical parametric oscillators as a coherent Ising machine," Nat. Photonics, vol. 8, pp. 937-942, 2014.

[64] N. G. Berloff, M. Silva, K. Kalinin, et al., "Realizing the classical XY Hamiltonian in polariton simulators," Nat. Mater., vol. 16, pp. 1120-1126, 2017.

[65] M. Nixon, E. Ronen, A. A. Friesem, and N. Davidson, “Observing geometric frustration with thousands of coupled lasers," Phys. Rev. Lett., vol. 110, p. 184102, 2013.

[66] M. Parto, W. Hayenga, A. Marandi, D. N. Christodoulides, and M. Khajavikhan, "Realizing spin Hamiltonians in nanoscale active photonic lattices," Nat. Mater., vol. 19, pp. 725-731, 2020.

[67] M. Parto, W. E. Hayenga, A. Marandi, D. N. Christodoulides, and M. Khajavikhan, "Nanolaser-based emulators of spin Hamiltonians,” Nanophotonics, vol. 1, 2020. https://doi.org/10. 1515/nanoph-2020-0230.

[68] R. El-Ganainy, L. Ge, M. Khajavikhan, and D. N. Christodoulides, “Supersymmetric laser arrays," Phys. Rev. A, vol. 92, p. 033818, 2015.

[69] M. P. Hokmabadi, N. S. Nye, R. El-Ganainy, D. N. Christodoulides, and M. Khajavikhan, "Supersymmetric laser arrays," Science, vol. 363, pp. 623-626, 2019.

[70] P. Miao, Z. Zhang, J. Sun, et al., "Orbital angular momentum microlaser,” Science, vol. 353, pp. 464-467, 2016.

[71] J. Ren, Y. G. N. Liu, M. Parto, et al., "Unidirectional light emission in PT-symmetric microring lasers," Opt. Express, vol. 26, pp. 27153-27160, 2018.

[72] W. E. Hayenga, M. Parto, J. Ren, et al., "Direct generation of tunable orbital angular momentum beams in microring lasers with broadband exceptional points," ACS Photonics, vol. 6, pp. 1895-1901, 2019.

[73] Y. D. Chong, L. Ge, H. Cao, and A. D. Stone, "Coherent perfect absorbers: time-reversed lasers,” Phys. Rev. Lett., vol. 105, p. 053901, 2010.

[74] Y. Sun, W. Tan, H. Li, J. Li, and H. Chen, “Experimental demonstration of a coherent perfect absorber with PT phase transition," Phys. Rev. Lett., vol. 112, p. 143903, 2014.

[75] S. Longhi, "PT-symmetric laser absorber," Phys. Rev. A, vol. 82, p. 031801, 2010.

[76] Z. J. Wong, Y.-L. Xu, J. Kim, et al., "Lasing and anti-lasing in a single cavity,” Nat. Photonics, vol. 10, pp. 796-801, 2016.

[77] Z. Lin, H. Ramezani, T. Eichelkraut, T. Kottos, H. Cao, and D. N. Christodoulides, "Unidirectional invisibility induced by PT-symmetric periodic structures," Phys. Rev. Lett., vol. 106, p. 213901, 2011.

[78] A. Regensburger, C. Bersch, M.-A. Miri, G. Onishchukov, D. N. Christodoulides, and U. Peschel, "Parity-time synthetic photonic lattices," Nature, vol. 488, pp. 167-171, 2012.
[79] M. Wimmer, M.-A. Miri, D. Christodoulides, and U. Peschel, "Observation of Bloch oscillations in complex PT-symmetric photonic lattices," Sci. Rep., vol. 5, p. 17760, 2015.

[80] L. Feng, Y.-L. Xu, W. S. Fegadolli, et al., “Experimental demonstration of a unidirectional reflectionless parity-time metamaterial at optical frequencies," Nat. Mater., vol. 12, pp. 108-113, 2013.

[81] L. Feng, X. Zhu, S. Yang, et al., "Demonstration of a large-scale optical exceptional point structure,” Opt. Express, vol. 22, pp. 1760-1767, 2014.

[82] Y. Yan and N. C. Giebink, "Passive PT symmetry in organic composite films via complex refractive index modulation," Adv. Opt. Mater., vol. 2, pp. 423-427, 2014.

[83] X. Zhu, L. Feng, P. Zhang, X. Yin, and X. Zhang, “One-way invisible cloak using parity-time symmetric transformation optics," Opt. Lett., vol. 38, pp. 2821-2824, 2013.

[84] D. L. Sounas, R. Fleury, and A. Alù, "Unidirectional cloaking based on metasurfaces with balanced loss and gain," Phys. Rev. Applied, vol. 4, p. 014005, 2015.

[85] K. G. Makris, Z. H. Musslimani, D. N. Christodoulides, and S. Rotter, "Constant-intensity waves and their modulation instability in non-Hermitian potentials," Nat. Commun., vol. 6, p. 7257, 2015.

[86] K. G. Makris, A. Brandstötter, P. Ambichl, Z. H. Musslimani, and S. Rotter, "Wave propagation through disordered media without backscattering and intensity variations," Light Sci. Appl., vol. 6, p. e17035, 2017.

[87] S. Longhi, "Bloch oscillations in complex crystals with PT symmetry," Phys. Rev. Lett., vol. 103, p. 123601, 2009.

[88] A. Szameit, M. C. Rechtsman, O. Bahat-Treidel, and M. Segev, "PT-symmetry in honeycomb photonic lattices," Phys. Rev. A, vol. 84, p. 021806, 2011.

[89] H. Ramezani, D. N. Christodoulides, V. Kovanis, I. Vitebskiy, and T. Kottos, “PT-symmetric Talbot effects," Phys. Rev. Lett., vol. 109, p. 033902, 2012.

[90] D. M. Jović, C. Denz, and M. R. Belić, "Anderson localization of light in PT-symmetric optical lattices,” Opt. Lett., vol. 37, pp. 4455-4457, 2012.

[91] R. Fleury, D. L. Sounas, and A. Alù, "Negative refraction and planar focusing based on parity-time symmetric metasurfaces," Phys. Rev. Lett., vol. 113, p. 023903, 2014.

[92] F. Monticone, C. A. Valagiannopoulos, and A. Alù, "Parity-time symmetric nonlocal metasurfaces: all-angle negative refraction and volumetric imaging," Phys. Rev. X, vol. 6, p. 041018, 2016.

[93] N. Lazarides and G. P. Tsironis, "Gain-driven discrete breathers in PT-symmetric nonlinear metamaterials," Phys. Rev. Lett., vol. 110, p. 053901, 2013.

[94] M. Lawrence, N. Xu, X. Zhang, et al., “Manifestation of PT symmetry breaking in polarization space with terahertz metasurfaces," Phys. Rev. Lett., vol. 113, p. 093901, 2014.

[95] N. S. Nye, A. E. Halawany, C. Markos, M. Khajavikhan, and D. N. Christodoulides, "Flexible PT-symmetric optical metasurfaces," Phys. Rev. Applied, vol. 13, p. 064005, 2020.

[96] Y. Lumer, Y. Plotnik, M. C. Rechtsman, and M. Segev, “Nonlinearly induced PT transition in photonic systems," Phys. Rev. Lett., vol. 111, p. 263901, 2013.

[97] A. U. Hassan, H. Hodaei, M.-A. Miri, M. Khajavikhan, and D. N. Christodoulides, "Nonlinear reversal of the PT-symmetric 
phase transition in a system of coupled semiconductor microring resonators," Phys. Rev. A, vol. 92, p. 063807, 2015.

[98] L. Ge and R. El-Ganainy, "Nonlinear modal interactions in parity-time (PT) symmetric lasers," Sci. Rep., vol. 6, p. 24889, 2016.

[99] S. Assawaworrarit, X. Yu, and S. Fan, "Robust wireless power transfer using a nonlinear parity-time-symmetric circuit," Nature, vol. 546, pp. 387-390, 2017.

[100] T. Wasak, P. Szańkowski, V. V. Konotop, and M. Trippenbach, "Four-wave mixing in a parity-time (PT)-symmetric coupler," Opt. Lett., vol. 40, pp. 5291-5294, 2015.

[101] R. El-Ganainy, J. I. Dadap, and R. M. Osgood, “Optical parametric amplification via non-Hermitian phase matching," Opt. Lett., vol. 40, pp. 5086-5089, 2015.

[102] A. A. Sukhorukov, Z. Xu, and Y. S. Kivshar, "Nonlinear suppression of time reversals in PT-symmetric optical couplers," Phys. Rev. A, vol. 82, p. 043818, 2010.

[103] D. A. Antonosyan, A. S. Solntsev, and A. A. Sukhorukov, "Paritytime anti-symmetric parametric amplifier," Opt. Lett., vol. 40, p. 4575, 2015.

[104] M.-A. Miri and A. Alù, "Nonlinearity-induced PT-symmetry without material gain,” New J. Phys., vol. 18, p. 065001, 2016.

[105] M. J. Ablowitz and Z. H. Musslimani, "Integrable nonlocal nonlinear schrodinger equation," Phys. Rev. Lett., vol. 110, p. 064105, 2013.

[106] N. V. Alexeeva, I. V. Barashenkov, A. A. Sukhorukov, and Y. S. Kivshar, "Optical solitons in PT-symmetric nonlinear couplers with gain and loss," Phys. Rev. A, vol. 85, p. 063837 , 2012.

[107] Y. V. Kartashov, B. A. Malomed, and L. Torner, “Unbreakable PT symmetry of solitons supported by inhomogeneous defocusing nonlinearity," Opt. Lett., vol. 39, pp. 5641-5644, 2014.

[108] V. V. Konotop, J. Yang, and D. A. Zezyulin, "Nonlinear waves in PT-symmetric systems,” Rev. Mod. Phys., vol. 88, p. 035002, 2016.

[109] H. Ramezani, T. Kottos, R. El-Ganainy, and D. N. Christodoulides, "Unidirectional nonlinear PT-symmetric optical structures,” Phys. Rev. A, vol. 82, p. 043803, 2010.

[110] P. Aleahmad, M. Khajavikhan, D. Christodoulides, and P. LiKamWa, “Integrated multi-port circulators for unidirectional optical information transport," Sci. Rep., vol. 7, p. 2129, 2017.

[111] N. Moiseyev, Non-Hermitian Quantum Mechanics, Cambridge, Cambridge University Press, 2011.

[112] M. H. Teimourpour, R. El-Ganainy, A. Eisfeld, A. Szameit, and D. N. Christodoulides, "Light transport in PT-invariant photonic structures with hidden symmetries," Phys. Rev. A, vol. 90, p. 053817, 2014.

[113] D. K. Armani, T. J. Kippenberg, S. M. Spillane, and K. J. Vahala, "Ultra-high-Q toroid microcavity on a chip," Nature, vol. 421, pp. 925-928, 2003.

[114] V. R. Almeida, C. A. Barrios, R. R. Panepucci, and M. Lipson, "All-optical control of light on a silicon chip," Nature, vol. 431, pp. 1081-1084, 2004.

[115] J.Zhu, Ş. K. Özdemir, L. He, and L. Yang, "Controlled manipulation of mode splitting in an optical microcavity by two Rayleigh scatterers," Opt. Express, vol. 18, pp. 23535-23543, 2010.

[116] J. Wiersig, "Structure of whispering-gallery modes in optical microdisks perturbed by nanoparticles," Phys. Rev. A, vol. 84, p. 063828, 2011.
[117] J. Wiersig, "Enhancing the sensitivity of frequency and energy splitting detection by using exceptional points: application to microcavity sensors for single-particle detection," Phys. Rev. Lett., vol. 112, p. 203901, 2014.

[118] H. Hodaei, A. U. Hassan, S. Wittek, et al., "Enhanced sensitivity at higher-order exceptional points," Nature, vol. 548, pp. 187-191, 2017.

[119] W. Chen, Ş. Kaya Özdemir, G. Zhao, J. Wiersig, and L. Yang, "Exceptional points enhance sensing in an optical microcavity," Nature, vol. 548, pp. 192-196, 2017.

[120] J. Ren, H. Hodaei, G. Harari, et al., "Ultrasensitive micro-scale parity-time-symmetric ring laser gyroscope," Opt. Lett., vol. 42, pp. 1556-1559, 2017.

[121] M. P. Hokmabadi, A. Schumer, D. N. Christodoulides, and M. Khajavikhan, "Non-Hermitian ring laser gyroscopes with enhanced Sagnac sensitivity," Nature, vol. 576, pp. 70-74, 2019.

[122] Y.-H. Lai, Y.-K. Lu, M.-G. Suh, Z. Yuan, and K. Vahala, "Observation of the exceptional-point-enhanced Sagnac effect," Nature, vol. 576, pp. 65-69, 2019.

[123] T. Goldzak, A. A. Mailybaev, and N. Moiseyev, "Light stops at exceptional points,” Phys. Rev. Lett., vol. 120, p. 013901, 2018.

[124] Q. Zhong, D. N. Christodoulides, M. Khajavikhan, K. G. Makris, and R. El-Ganainy, "Power-law scaling of extreme dynamics near higher-order exceptional points,” Phys. Rev. A, vol. 97, p. 020105, 2018.

[125] A. Pick, B. Zhen, O. D. Miller, et al., "General theory of spontaneous emission near exceptional points," Opt. Express, vol. 25, pp. 12325-12348, 2017.

[126] Z. Lin, A. Pick, M. Lončar, and A. W. Rodriguez, “Enhanced spontaneous emission at third-order Dirac exceptional points in inverse-designed photonic crystals," Phys. Rev. Lett., vol. 117, p. 107402, 2016.

[127] Q. Zhong, J. Ren, M. Khajavikhan, D. N. Christodoulides, Ş. K. Özdemir, and R. El-Ganainy, "Sensing with exceptional surfaces in order to combine sensitivity with robustness," Phys. Rev. Lett., vol. 122, p. 153902, 2019.

[128] H. Zhou, J. Y. Lee, S. Liu, and B. Zhen, "Exceptional surfaces in PT-symmetric non-Hermitian photonic systems," Optica, vol. 6, pp. 190-193, 2019.

[129] W. Langbein, "No exceptional precision of exceptional-point sensors," Phys. Rev. A, vol. 98, p. 023805, 2018.

[130] H. Wang, Y.-H. Lai, Z. Yuan, M.-G. Suh, and K. Vahala, "Petermann-factor sensitivity limit near an exceptional point in a Brillouin ring laser gyroscope," Nat. Commun., vol. 11, p. 1610, 2020.

[131] N. A. Mortensen, P. a. D. Gonçalves, M. Khajavikhan, D. N. Christodoulides, C. Tserkezis, and C. Wolff, "Fluctuations and noise-limited sensing near the exceptional point of paritytime-symmetric resonator systems," Optica, vol. 5, pp. 1342-1346, 2018.

[132] M. Zhang, W. Sweeney, C. W. Hsu, L. Yang, A. D. Stone, and L. Jiang, "Quantum noise theory of exceptional point amplifying sensors," Phys. Rev. Lett., vol. 123, p. 180501, 2019.

[133] M. V. Berry, "Quantal phase factors accompanying adiabatic changes," Proc. R. Soc. Lond. A Math. Phys. Sci., vol. 392, pp. 45-57, 1984.

[134] W. D. Heiss, "The physics of exceptional points," J. Phys. A Math. Theor., vol. 45, p. 444016, 2012. 
[135] A. A. Mailybaev, O. N. Kirillov, and A. P. Seyranian, "Geometric phase around exceptional points," Phys. Rev. A, vol. 72, p. 014104, 2005.

[136] C. Dembowski, H.-D. Gräf, H. L. Harney, et al., "Experimental observation of the topological structure of exceptional points," Phys. Rev. Lett., vol. 86, pp. 787-790, 2001.

[137] S.-B. Lee, J. Yang, S. Moon, et al., "Observation of an exceptional point in a chaotic optical microcavity," Phys. Rev. Lett., vol. 103, p. 134101, 2009.

[138] T. Gao, E. Estrecho, K. Y. Bliokh, et al., "Observation of nonHermitian degeneracies in a chaotic exciton-polariton billiard," Nature, vol. 526, pp. 554-558, 2015.

[139] R. Uzdin, A. Mailybaev, and N. Moiseyev, "On the observability and asymmetry of adiabatic state flips generated by exceptional points," J. Phys. A Math. Theor., vol. 44, p. 435302, 2011.

[140] T. J. Milburn, J. Doppler, C. A. Holmes, S. Portolan, S. Rotter, and P. Rabl, "General description of quasiadiabatic dynamical phenomena near exceptional points," Phys. Rev. A, vol. 92, p. 052124, 2015.

[141] J. Doppler, A. A. Mailybaev, J. Böhm, et al., "Dynamically encircling an exceptional point for asymmetric mode switching," Nature, vol. 537, pp. 76-79, 2016.

[142] H. Xu, D. Mason, L. Jiang, and J. G. E. Harris, "Topological energy transfer in an optomechanical system with exceptional points," Nature, vol. 537, pp. 80-83, 2016.

[143] J. W. Yoon, Y. Choi, C. Hahn, et al., "Time-asymmetric loop around an exceptional point over the full optical communications band," Nature, vol. 562, pp. 86-90, 2018.

[144] A. U. Hassan, B. Zhen, M. Soljačić, M. Khajavikhan, and D. N. Christodoulides, "Dynamically encircling exceptional points: exact evolution and polarization state conversion," Phys. Rev. Lett., vol. 118, p. 093002, 2017.

[145] A. U. Hassan, G. L. Galmiche, G. Harari, et al., "Chiral state conversion without encircling an exceptional point," Phys. Rev. A, vol. 96, p. 052129, 2017.

[146] Q. Zhong, M. Khajavikhan, D. N. Christodoulides, and R. ElGanainy, "Winding around non-Hermitian singularities," Nat. Commun., vol. 9, p. 4808, 2018.

[147] H. Zhou, C. Peng, Y. Yoon, et al., "Observation of bulk Fermi arc and polarization half charge from paired exceptional points," Science, vol. 359, pp. 1009-1012, 2018.

[148] E. J. Bergholtz and J. C. Budich, "Non-Hermitian Weyl physics in topological insulator ferromagnet junctions," Phys. Rev. Research, vol. 1, p. 012003, 2019.

[149] X.-X. Zhang and M. Franz, "Non-Hermitian exceptional Landau quantization in electric circuits," Phys. Rev. Lett., vol. 124, p. $046401,2020$.

[150] B. Zhen, C. W. Hsu, Y. Igarashi, et al., "Spawning rings of exceptional points out of Dirac cones," Nature, vol. 525, pp. 354-358, 2015.

[151] J. C. Budich, J. Carlström, F. K. Kunst, and E. J. Bergholtz, "Symmetry-protected nodal phases in non-Hermitian systems," Phys. Rev. B, vol. 99, p. 041406, 2019.

[152] R. Okugawa and T. Yokoyama, "Topological exceptional surfaces in non-Hermitian systems with parity-time and parity-particlehole symmetries," Phys. Rev. B, vol. 99, p. 041202, 2019.

[153] T. Yoshida, R. Peters, N. Kawakami, and Y. Hatsugai, "Symmetry-protected exceptional rings in two-dimensional correlated systems with chiral symmetry," Phys. Rev. B, vol. 99, p. 121101, 2019

[154] X. Wan, A. M. Turner, A. Vishwanath, and S. Y. Savrasov, "Topological semimetal and Fermi-arc surface states in the electronic structure of pyrochlore iridates," Phys. Rev. B, vol. 83, p. 205101, 2011.

[155] Y. Xu, S.-T. Wang, and L.-M. Duan, "Weyl exceptional rings in a three-dimensional dissipative cold atomic gas," Phys. Rev. Lett., vol. 118, p. 045701, 2017.

[156] A. Cerjan, S. Huang, M. Wang, K. P. Chen, Y. Chong, and M. C. Rechtsman, "Experimental realization of a Weyl exceptional ring," Nat. Photonics, vol. 13, pp. 623-628, 2019.

[157] K. V. Klitzing, G. Dorda, and M. Pepper, "New method for highaccuracy determination of the fine-structure constant based on quantized Hall resistance," Phys. Rev. Lett., vol. 45, pp. 494-497, 1980.

[158] C. L. Kane and E. J. Mele, "Quantum spin Hall effect in graphene,” Phys. Rev. Lett., vol. 95, p. 226801, 2005.

[159] B. A. Bernevig, T. L. Hughes, and S.-C. Zhang, "Quantum spin Hall effect and topological phase transition in HgTe quantum wells," Science, vol. 314, pp. 1757-1761, 2006.

[160] M. König, S. Wiedmann, C. Brüne, et al., "Quantum spin Hall insulator state in HgTe quantum wells," Science, vol. 318, pp. 766-770, 2007.

[161] F. D. M. Haldane and S. Raghu, "Possible realization of directional optical waveguides in photonic crystals with broken time-reversal symmetry," Phys. Rev. Lett., vol. 100, p. 013904, 2008.

[162] Z. Wang, Y. Chong, J. D. Joannopoulos, and M. Soljačić, "Observation of unidirectional backscattering-immune topological electromagnetic states," Nature, vol. 461, pp. 772-775, 2009.

[163] M. Hafezi, E. A. Demler, M. D. Lukin, and J. M. Taylor, "Robust optical delay lines with topological protection,” Nat. Phys., vol. 7, pp. 907-912, 2011.

[164] A. B. Khanikaev, S. Hossein Mousavi, W.-K. Tse, M. Kargarian, A. H. MacDonald, and G. Shvets, "Photonic topological insulators," Nat. Mater., vol. 12, pp. 233-239, 2013.

[165] T. Ozawa, H. M. Price, A. Amo, et al., "Topological photonics," Rev. Mod. Phys., vol. 91, p. 015006, 2019.

[166] Y. Ota, K. Takata, T. Ozawa, et al., "Active topological photonics," Nanophotonics, vol. 9, pp. 547-567, 2020.

[167] W. P. Su, J. R. Schrieffer, and A. J. Heeger, "Solitons in polyacetylene,” Phys. Rev. Lett., vol. 42, pp. 1698-1701, 1979.

[168] P. St-Jean, V. Goblot, E. Galopin, et al., "Lasing in topological edge states of a one-dimensional lattice," Nat. Photonics, vol. 11, pp. 651-656, 2017.

[169] M. Parto, S. Wittek, H. Hodaei, et al., "Edge-mode lasing in 1D topological active arrays," Phys. Rev. Lett., vol. 120, p. 113901, 2018.

[170] H. Zhao, P. Miao, M. H. Teimourpour, et al., "Topological hybrid silicon microlasers," Nat. Commun., vol. 9, p. 981, 2018.

[171] Y. Ota, R. Katsumi, K. Watanabe, S. Iwamoto, and Y. Arakawa, "Topological photonic crystal nanocavity laser," Commun. Phys., vol. 1, pp. 1-8, 2018.

[172] S. Malzard, C. Poli, and H. Schomerus, "Topologically protected defect states in open photonic systems with non-Hermitian charge-conjugation and parity-time symmetry," Phys. Rev. Lett., vol. 115, p. 200402, 2015. 
[173] G. Harari, M. A. Bandres, Y. Lumer, et al., "Topological insulator laser: theory," Science, vol. 359, 2018. https://doi.org/10. 1126/science.aar4003.

[174] M. A. Bandres, S. Wittek, G. Harari, et al., "Topological insulator laser: Experiments,” Science, vol. 359, 2018. https://doi.org/ 10.1126/science.aar4005.

[175] B. Bahari, A. Ndao, F. Vallini, A. E. Amili, Y. Fainman, and B. Kanté, "Nonreciprocal lasing in topological cavities of arbitrary geometries," Science, vol. 358, pp. 636-640, 2017.

[176] S. Klembt, T. H. Harder, O. A. Egorov, et al., "Exciton-polariton topological insulator," Nature, vol. 562, pp. 552-556, 2018.

[177] T. Ma and G. Shvets, "All-Si valley-Hall photonic topological insulator,” New J. Phys., vol. 18, p. 025012, 2016.

[178] Y. Zeng, U. Chattopadhyay, B. Zhu, et al., "Electrically pumped topological laser with valley edge modes," Nature, vol. 578, pp. 246-250, 2020.

[179] H. Zhong, Y. Li, D. Song. et al., "Topological valley hall edge state lasing," arXiv:1912.13003 [nlin, physics:physics], 2019.

[180] Z.-K. Shao, H.-Z. Chen, S. Wang, et al., "A high-performance topological bulk laser based on band-inversion-induced reflection," Nat. Nanotechnol., vol. 15, pp. 67-72, 2020.

[181] F. D. M. Haldane, "Model for a quantum Hall effect without Landau levels: condensed-matter realization of the "parity anomaly,"” Phys. Rev. Lett., vol. 61, pp. 2015-2018, 1988.

[182] S. Weimann, M. Kremer, Y. Plotnik, et al., “Topologically protected bound states in photonic parity-time-symmetric crystals," Nat. Mater., vol. 16, pp. 433-438, 2017.

[183] J. M. Zeuner, M. C. Rechtsman, Y. Plotnik, et al., "Observation of a topological transition in the bulk of a non-Hermitian system," Phys. Rev. Lett., vol. 115, p. 040402, 2015.

[184] L. Jin, P. Wang, and Z. Song, "Su-Schrieffer-Heeger chain with one pair of PT-symmetric defects," Sci. Rep., vol. 7, p. 5903, 2017.

[185] K. Takata and M. Notomi, "Photonic topological insulating phase induced solely by gain and loss," Phys. Rev. Lett., vol. 121, p. 213902, 2018.

[186] S. Liu, S. Ma, C. Yang, et al., "Gain- and loss-induced topological insulating phase in a non-Hermitian electrical circuit," Phys. Rev. Applied, vol. 13, p. 014047, 2020.

[187] A. K. Harter, T. E. Lee, and Y. N. Joglekar, “PT-breaking threshold in spatially asymmetric Aubry-André and Harper models: hidden symmetry and topological states," Phys. Rev. A, vol. 93, p. 062101, 2016.

[188] L. Xiao, X. Zhan, Z. H. Bian, et al., "Observation of topological edge states in parity-time-symmetric quantum walks," Nat. Phys., vol. 13, pp. 1117-1123, 2017.

[189] L. He, Z. Addison, J. Jin, E. J. Mele, S. G. Johnson, and B. Zhen, "Floquet Chern insulators of light," Nat. Commun., vol. 10, p. 4194, 2019.

[190] M. Li, X. Ni, M. Weiner, A. Alù, and A. B. Khanikaev, “Topological phases and nonreciprocal edge states in non-Hermitian Floquet insulators," Phys. Rev. B, vol. 100, p. 045423, 2019.

[191] Y. G. N. Liu, P. Jung, M. Parto, J. Leshin, D. N. Christodoulides, and M. Khajavikhan, "Towards a non-magnetic topological Haldane laser," in Conference on Lasers and Electro-Optics (2019), Paper FW3D.1, Optical Society of America, 2019, p. FW3D.1.

[192] C. Poli, M. Bellec, U. Kuhl, F. Mortessagne, and H. Schomerus, "Selective enhancement of topologically induced interface states in a dielectric resonator chain," Nat. Commun., vol. 6, p. $6710,2015$.

[193] R. El-Ganainy and M. Levy, "Optical isolation in topologicaledge-state photonic arrays," Opt. Lett., vol. 40, pp. 5275-5278, 2015.

[194] E. Makri, R. Thomas, and T. Kottos, "Reflective limiters based on self-induced violation of PT symmetry," Phys. Rev. A, vol. 97, p. 043864, 2018.

[195] H. Zhao, X. Qiao, T. Wu, B. Midya, S. Longhi, and L. Feng, "NonHermitian topological light steering," Science, vol. 365, pp. 1163-1166, 2019.

[196] K. Kawabata, K. Shiozaki, M. Ueda, and M. Sato, "Symmetry and topology in non-Hermitian physics," Phys. Rev. X, vol. 9, p. 041015, 2019.

[197] H. Zhou and J. Y. Lee, "Periodic table for topological bands with non-Hermitian symmetries," Phys. Rev. B, vol. 99, p. 235112, 2019.

[198] A. P. Schnyder, S. Ryu, A. Furusaki, and A. W. W. Ludwig, "Classification of topological insulators and superconductors in three spatial dimensions," Phys. Rev. B, vol. 78, p. 195125, 2008.

[199] A. Kitaev, "Periodic table for topological insulators and superconductors," AIP Conf. Proc., vol. 1134, pp. 22-30, 2009.

[200] K. Kawabata, S. Higashikawa, Z. Gong, Y. Ashida, and M. Ueda, "Topological unification of time-reversal and particle-hole symmetries in non-Hermitian physics," Nat. Commun., vol. 10, p. 297, 2019.

[201] Y. Xiong, "Why does bulk boundary correspondence fail in some non-Hermitian topological models," J. Phys. Commun., vol. 2, p. 035043, 2018.

[202] T. E. Lee, “Anomalous edge state in a non-Hermitian lattice," Phys. Rev. Lett., vol. 116, p. 133903, 2016.

[203] H. Shen, B. Zhen, and L. Fu, "Topological band theory for nonHermitian Hamiltonians," Phys. Rev. Lett., vol. 120, p. 146402, 2018.

[204] L. Jin and Z. Song, "Bulk-boundary correspondence in a nonHermitian system in one dimension with chiral inversion symmetry,” Phys. Rev. B, vol. 99, p. 081103, 2019.

[205] S. Yao and Z. Wang, "Edge states and topological invariants of non-Hermitian systems," Phys. Rev. Lett., vol. 121, p. 086803 , 2018.

[206] S. Yao, F. Song, and Z. Wang, "Non-Hermitian chern bands," Phys. Rev. Lett., vol. 121, p. 136802, 2018.

[207] N. Okuma, K. Kawabata, K. Shiozaki, and M. Sato, "Topological origin of non-Hermitian skin effects," Phys. Rev. Lett., vol. 124, p. 086801, 2020.

[208] K. Kawabata, K. Shiozaki, and M. Ueda, "Anomalous helical edge states in a non-Hermitian Chern insulator," Phys. Rev. B, vol. 98, p. 165148, 2018.

[209] S. Weidemann, M. Kremer, T. Helbig, et al., "Topological funneling of light," Science, vol. 368, pp. 311-314, 2020.

[210] Z. Gong, Y. Ashida, K. Kawabata, K. Takasan, S. Higashikawa, and M. Ueda, "Topological phases of non-Hermitian systems," Phys. Rev. X, vol. 8, p. 031079, 2018.

[211] K. Yokomizo and S. Murakami, "Non-bloch band theory of nonHermitian systems," Phys. Rev. Lett., vol. 123, p. 066404, 2019.

[212] D. Leykam, K. Y. Bliokh, C. Huang, Y. D. Chong, and F. Nori, "Edge modes, degeneracies, and topological numbers in nonHermitian systems," Phys. Rev. Lett., vol. 118, p. 040401, 2017. 
[213] F. K. Kunst, E. Edvardsson, J. C. Budich, and E. J. Bergholtz, "Biorthogonal bulk-boundary correspondence in nonHermitian systems," Phys. Rev. Lett., vol. 121, p. 026808, 2018.

[214] K.-I. Imura and Y. Takane, "Generalized bulk-edge correspondence for non-Hermitian topological systems," Phys. Rev. B, vol. 100, p. 165430, 2019.

[215] W. A. Benalcazar, B. A. Bernevig, and T. L. Hughes, "Quantized electric multipole insulators," Science, vol. 357, pp. 61-66, 2017.

[216] W. A. Benalcazar, B. A. Bernevig, and T. L. Hughes, "Electric multipole moments, topological multipole moment pumping, and chiral hinge states in crystalline insulators," Phys. Rev. B, vol. 96, p. 245115, 2017.

[217] F. Schindler, A. M. Cook, M. G. Vergniory, et al., "Higher-order topological insulators,” Sci. Adv., vol. 4, p. eaat0346, 2018.
[218] C. H. Lee, L. Li, and J. Gong, "Hybrid higher-order skintopological modes in nonreciprocal systems," Phys. Rev. Lett., vol. 123, p. 016805, 2019.

[219] X.-W. Luo and C. Zhang, "Higher-order topological corner states induced by gain and loss," Phys. Rev. Lett., vol. 123, p. 073601, 2019.

[220] M. Ezawa, "Non-Hermitian higher-order topological states in nonreciprocal and reciprocal systems with their electric-circuit realization,” Phys. Rev. B, vol. 99, p. 201411, 2019.

[221] T. Liu, Y.-R. Zhang, Q. Ai, et al., "Second-order topological phases in non-Hermitian systems," Phys. Rev. Lett., vol. 122, p. 076801, 2019.

[222] Z. Zhang, M. Rosendo López, Y. Cheng, X. Liu, and J. Christensen, "Non-Hermitian sonic second-order topological insulator," Phys. Rev. Lett., vol. 122, p. 195501, 2019. 